\title{
Evaluation of methods for the inference of ancestral recombination graphs
}

\author{
Débora Y. C. Brandt*,1, Xinzhu Wei ${ }^{\dagger}$, Yun Deng ${ }^{\ddagger}$, Andrew H. Vaughn ${ }^{\ddagger}$ and Rasmus Nielsen*, ${ }^{*}, 1$ \\ *Department of Integrative Biology, University of California Berkeley, Berkeley, CA 94720, USA, ${ }^{\dagger}$ Department of Computer Science, University of California Los \\ Angeles, Los Angeles, 90095, CA, USA, ${ }^{\ddagger}$ Center for Computational Biology, University of California, Berkeley, CA 94720, USA, ${ }^{\S}$ Department of Statistics, \\ University of California Berkeley, Berkeley, CA 94720, USA, GLOBE Institute, University of Copenhagen, Oester Voldgade 5-7 1350 Copenhagen K, Denmark
}

\begin{abstract}
The ancestral recombination graph (ARG) is a structure that describes the joint genealogies of sampled DNA sequences along the genome. Recent computational methods have made impressive progress towards scalably estimating whole-genome genealogies. In addition to inferring the ARG, some of these methods can also provide ARGs sampled from a defined posterior distribution. Obtaining good samples of ARGs is crucial for quantifying statistical uncertainty and for estimating population genetic parameters such as effective population size, mutation rate, and allele age. Here, we use simulations to benchmark three popular ARG inference programs: ARGweaver, Relate, and tsdate. We use neutral coalescent simulations to 1) compare the true coalescence times to the inferred times at each locus; 2) compare the distribution of coalescence times across all loci to the expected exponential distribution; 3) evaluate whether the sampled coalescence times have the properties expected of a valid posterior distribution. We find that inferred coalescence times at each locus are more accurate in ARGweaver and Relate than in tsdate. However, all three methods tend to overestimate small coalescence times and underestimate large ones. Lastly, the posterior distribution of ARGweaver is closer to the expected posterior distribution than Relate's, but this higher accuracy comes at a substantial trade-off in scalability. The best choice of method will depend on the number and length of input sequences and on the goal of downstream analyses, and we provide guidelines for the best practices.
\end{abstract}

KEYWORDS Ancestral recombination graph; ARGweaver; Relate; tsdate; simulation; calibration

The ancestral recombination graph (ARG) is a representation of the genealogies of sampled DNA sequences as a set of coalescence and recombination events (Griffiths and Marjoram 1997). Hudson (1983) first proposed a model that combines recombination and coalescence to describe genealogies. At each given site, the genealogy can be described by the single-locus coalescent model (Kingman 1982), but because recombination breaks loci apart, the local genealogies can differ between sites (Figure 1A).

\footnotetext{
${ }^{1}$ University of California, Berkeley Department of Integrative Biology 3040 Valley Life Sciences Building \#3140 Berkeley, CA 94720-3140. deboraycb@berkeley.edu, rasmus_nielsen@berkeley.edu
}

\section{The ARG}

An ARG can be represented as a sequence of marginal coalescence trees (Figure 1C). In some representations, the sequence of trees may or may not contain the full information of the ARG, depending on whether the times of recombination events are stored with the trees (Rasmussen et al. 2014). Furthermore, if only topology changing recombination events are represented, information regarding recombination events that do not lead to topology changes are lost (Kelleher et al. 2019). Finally, some representations of tree sequences allow more than one recombination event between trees (Speidel et al. 2019). In the latter two cases, each tree will potentially be an average of 

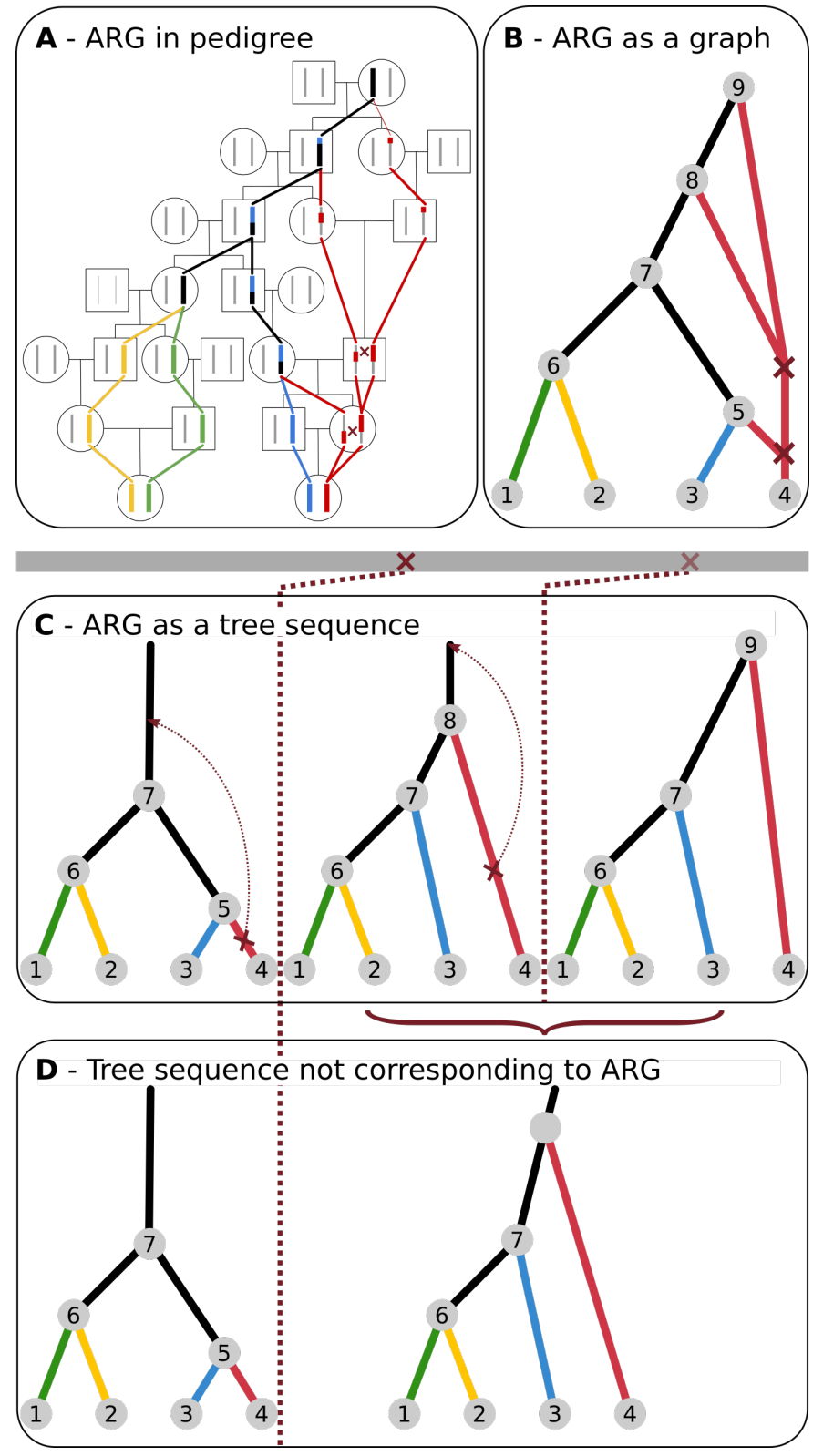

Figure 1 Schematic representations of the genealogy of a sample of two diploid individuals. Colors denote the four haplotypes sampled, and black lines indicate lineages or sequence tracts where at least one coalescence has occurred. Dark red crosses indicate recombination events. (A) The genealogy embedded in a pedigree. (B) An ancestral recombination graph (ARG) that fully represents all genealogical relationships shown in A, assuming that recombination events are annotated with the sequence coordinates. (C) An equivalent representation of the ARG as a sequence of local trees separated by a single recombination event. (D) A tree sequence that does not correspond to the full ARG. Instead, the second tree is an average of the
Figure 1 Caveats: 1) the pedigree representation shows a large amount of inbreeding (i.e. very small effective population size), so the coalescent would not be the best model for it. The pedigree is used here to explicitly show the process of coalescence as lineages merging and recombination as lineages splitting, as we move backwards in time. 2) part $\mathrm{D}$ shows a tree sequence with a missing recombination. This could happen in ARG inference because that particular recombination does not change the tree topology. Another scenario where tree sequences do not correspond to an ARG is when two consecutive trees are separated by more than one topology-changing recombination event. In those cases, some sort of average tree for that position would likely be inferred.

multiple coalescence trees. Figure 1D shows an example of a tree sequence that does not correspond to the underlying full ARG, since one of its local trees is an average of two adjacent trees with identical topologies. Sequences of trees can be represented efficiently in computer memory by noting that a single node is part of many marginal trees (Kelleher et al. 2018).

The full ARG contains all the information in a sample of DNA sequences regarding demography. Specifically, for a set of demographic parameters $\theta$, parameters of the mutational process $\mu$, sequence data $x$, and ARG $G, p(x \mid \theta, \mu, G)=p(x \mid \mu, G)$, i.e. if $G$ is known there is no more information in the data about $\theta$. A similar statement can be made for recombination and selection, if the leaf nodes of $G$ are augmented with the allelic state at the selected loci. Therefore, the ARG is necessarily as least as informative as the combination of any and all summary statistics traditionally used to infer evolutionary processes (such as $F_{S T}, \pi$, Tajima's $D$, or EHH). Knowledge of the ARG is key for constructing powerful methods for extracting population genetic information from DNA sequencing data.

\section{Inferring ARGs}

Unfortunately, ARGs cannot be directly observed but must be inferred from the data. Together with an estimate of the ARG, it is desirable to quantify the uncertainty around the inferred ARG, for example by obtaining samples of ARGs according to their posterior probabilities. Such samples can be used local trees at that region.

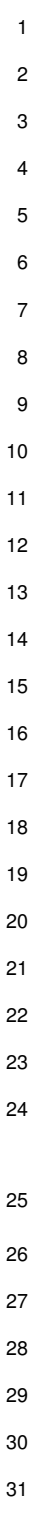


to quantify uncertainty regarding ARG inferences in downstream analyses. Accurate sampling from the posterior distribution is especially relevant for downstream methods that rely on importance sampling to infer evolutionary parameters from ARGs. In essence, these methods weight parameter inference under each sampled ARG by the ARG probability and therefore require that the samples of ARGs accurately reflect their probability distribution. These types of methods can be used to infer population size history, selection (Stern et al. 2019), migration (Osmond and Coop 2021), mutation rates and recombination rates.

Inferring full ARGs and quantifying inference uncertainty by sampling from the posterior distribution is a challenging problem computationally. It requires navigating a high-dimensional distribution of ARGs, which are themselves a complicated data structure. For this reason, inferring ARGs and sampling from their posterior distribution seemed like a nearly impossible endeavour some years ago, but important methodological developments now allow us to do so. Today, there are several methods available to estimate the ARG or a tree sequence approximation of the ARG, including ARGweaver (Rasmussen et al. 2014), Relate (Speidel et al. 2019) and tsinfer/tsdate (Kelleher et al. 2019; Wohns et al. 2021).

\section{Approximations of the ARG}

The classical way to include recombination in coalescence models is to consider the temporal process of lineage splitting caused by recombination and lineage merger caused by coalescences as one moves backwards in time (Hudson 1983; Griffiths and Marjoram 1997) (Figure 1A,B). Wiuf and Hein (1999) considered instead the spatial process of recombination along a sequence. In this formulation, the ARG is constructed as a sequence of local coalescent trees along a genome, where each tree is separated from adjacent trees by recombination events (Figure 1C). At each recombination breakpoint, a new tree is formed from the immediately preceding tree. To form the next tree, first one of the branches in the current tree is detached. Next, a point earlier than the detachment point is randomly chosen from any of the branches in any of the previous trees in the sequence. Finally, the detached branch coalesces to this chosen point.

To improve the computational efficiency in simulations, McVean and Cardin (2005) proposed approximating the spatial process as a Markovian process called the Sequentially Markovian Coalescent (SMC). In the SMC, when a lineage is detached from a tree at a recombination event, it can only coalesce back to one of the other lineages present at the current tree. Marjoram and Wall (2006) proposed an improved approximation, the $\mathrm{SMC}^{\prime}$, in which the detached lineage can coalesce to any branch in the current tree, including the one it was detached from. This means that some recombination events in this model do not generate a different local coalescent tree. This simple modification significantly improves the model in terms of approximating the full coalescent (Marjoram and Wall 2006; Wilton et al. 2015).

A heuristic approximation to the coalescence with recombination proposed by Li and Stephens (2003), extending ideas from Stephens and Donnelly (2000), approximates the coalescent with recombination using a copying process where one sequence is modeled as a copy of other sequences in the sample, with errors representing mutations and switches in the copying template representing recombination events. While this representation has disadvantages, such as a dependence on the input order of sequences, it has proven computationally convenient for many purposes, including demography inference, introgression detection, and more (Sheehan et al. 2013; Steinrücken et al. 2018, 2019).

The formulation of the coalescent with recombination approximated as a Markovian process generating tree sequences in the SMC (McVean and Cardin 2005) and SMC' (Marjoram and Wall 2006) and as a copying process of individual sequences by $\mathrm{Li}$ and Stephens (2003), paved the way for more scalable ARG inference methods. Notably, ARGweaver (Rasmussen et al. 2014) based on the SMC or SMC' model, and Relate (Speidel et al. 2019) and tsinfer/tsdate (Kelleher et al. 2019; Wohns et al. 2021) based on the model by Li and Stephens (2003).

\section{ARGweaver}

ARGweaver uses Markov Chain Monte Carlo (MCMC) to sample ARGs from the posterior distribution under the SMC or SMC'. It relies on a discretization of time (such that all recombination and coalescence events are only allowed to happen at a discrete set of time points) which makes the state space of ARGs finite countable and allows the use of discrete state-space Hidden Markov Models (HMMs). It then uses a lineage threading approach, which is a Gibbs sampling update, to sample the history of a single lin- 
Table 1 Genome-wide genealogy inference programs compared.

\begin{tabular}{rcccccc}
\hline Program & $\begin{array}{c}\text { Samples } \\
\text { topologies }\end{array}$ & $\begin{array}{c}\text { Samples } \\
\text { coalescence } \\
\text { times }\end{array}$ & $\begin{array}{c}\text { Supports } \\
\text { demographic } \\
\text { model }\end{array}$ & $\begin{array}{c}\text { Scalability } \\
\text { (number of } \\
\text { genomes) }\end{array}$ & $\begin{array}{c}\text { Outputs } \\
\text { ARG }\end{array}$ & $\begin{array}{c}\text { Supports } \\
\text { unphased } \\
\text { data }\end{array}$ \\
\hline ARGweaver & Yes & Yes & No & $\sim 50$ & Yes & Yes \\
ARGweaver-D* & Yes & Yes & Yes & $\sim 50$ & Yes & Yes \\
Relate & No & Yes & No & $\sim 1 \mathrm{e} 3$ & No & No \\
\hline
\end{tabular}

* (Hubisz et al. 2020)

eage or haplotype from the full conditional posterior distribution given the rest of the ARG connecting all other haplotypes.

\section{Relate}

Relate simplifies the problem of ARG inference by inferring tree sequences, instead of full ARGs. Inference is divided into 2 steps. First, the Li and Stephens (2003) haplotype copying model is used to calculate pairwise distances between samples in order to infer local tree topologies. Next, it uses MCMC under a coalescent prior to infer coalescence times on those local trees. Relate is able to output samples of coalescence times from the posterior distribution using this MCMC approach, but it does so for the same fixed sequence of tree topologies. This is different from the ARGweaver MCMC sampling, which also samples the tree topology space (Table 1).

\section{tsinfer, tsdate, and the tree sequence framework}

Tsdate (Wohns et al. 2021) is a method that adds coalescence times to tree sequences inferred by tsinfer (Kelleher et al. 2019). Similarly to Relate, tsinfer is also based on the copying process from $\mathrm{Li}$ and Stephens (2003). A key innovation of tsinfer is a highly efficient tree sequence data structure which stores sequence data and genealogies (Kelleher et al. 2016, 2018, 2019; Ralph et al. 2020). tsinfer performs inference in two steps. First, it recreates ancestral haplotypes based on allele sharing between samples. Next, it uses an HMM to infer the closest matches between ancestral haplotypes and the sampled haplotypes using an ancestral copying process modified from the classical Li and Stephens (2003) model to generate the tree topology. Finally, nodes in the local tree topologies inferred by tsinfer can be dated by tsdate. tsdate uses a conditional coalescent prior, where the standard coalescent is conditioned on the number of descendants of each node on a local tree. Like ARGweaver, tsdate also discretizes time for computational efficiency. This framework infers a fixed topology and coalescence time, but it has the potential to sample coalescence times.

\section{Benchmarking of ARG inference methods}

Here, we use simulations to benchmark ARG inferences in ARGweaver (Rasmussen et al. 2014), Relate (Speidel et al. 2019), and tsdate (Wohns et al. 2021). We focus mainly on ARGweaver and Relate because they report measures of uncertainty in inference by allowing the user to output multiple samples from the posterior distribution. Sampling from the posterior is not currently implemented in tsdate (Table 1 ), but we include it in this evaluation because it is a promising framework for very fast tree-sequence inference, and it will likely provide an option to output samples from the posterior distribution of treesequences in future updates.

We run coalescent simulations on msprime (Kelleher et al. 2016) and compare the true (simulated) ARGs to the ARGs sampled by ARGweaver and tree sequences sampled by Relate and inferred by tsinfer/tsdate. We compare the ARGs based on their pairwise coalescence times using three different types of evaluation. First, we compare the true pairwise coalescence time at each site to the inferred time. Second, we compare the overall distribution of pairwise coalescence times across all sites and all MCMC 
samples to the expected distribution. In Bayesian inference, the data averaged posterior distribution is equal to the prior. Since data are simulated under the standard coalescent with recombination the data averaged posterior should be exponential with rate 1 in coalescence time units ( $2 N_{e}$ generations, where $N_{e}$ is the effective population size). Third, we used simulation-based calibration (SBC) (Cook et al. 2006; Talts et al. 2020) to evaluate if the posterior distributions sampled by ARGweaver and Relate are well calibrated (see details in Methods).

\section{Methods}

\section{Simulations}

We simulated tree sequences and SNP data with msprime version 0.7.4 (Kelleher et al. 2016). For simulations with Jukes and Cantor (1969) mutational model, we used msprime version 1.0.2 (Baumdicker et al. 2021) to add mutations to trees simulated under msprime 0.7.4. Unless otherwise noted, simulations were done under the standard neutral coalescent (Hudson model in msprime) and using the following parameters: 4 diploid samples (i.e. 8 haplotypes), effective population size of 10,000 diploids $\left(2 N_{e}=20,000\right)$, mutation rate and recombination rate of $2 \times 10^{-8}$ per base pair per generation and a total sequence length of $100 \mathrm{Mb}$.

We varied these standard simulation scenarios in several ways: using SMC and SMC' models, different numbers of samples (4, 16, 32 and 80 haplotypes), and a 10-fold increase in the mutation to recombination ratio (both by increasing the mutation rate to $2 \times 10^{-7}$ and by decreasing the recombination rate to $2 \times 10^{-9}$ ).

We ran msprime simulations using the python Application Programming Interface (API) and stored data in VCF and tree sequence format.

\section{ARGweaver}

VCF files from msprime were converted to ARGweaver sites format using a custom python script. We ran ARGweaver's arg-sample program to sample ARGs. This was done in parallel on $205 \mathrm{Mb}$ segments, using the -region option. We used the same values used in the msprime simulations (-mutrate and -recombrate 2e-8 and -popsize 10000) and except where otherwise noted, we ran ARGweaver using the SMC' model (-smcprime option). We ran ARGweaver with 1200 or 2200 iterations (-iters) (with burn-in of the first 200 or 1200 iterations, respectively), depending on how long it took to converge. Assessment of convergence is described below and in the Supplementary Materials, Evaluating MCMC Convergence. We extracted 100 MCMC samples from every 10th iteration among the last 1,000 iterations (default sample-step 10).

We extracted all pairwise coalescence times with the program arg-summarize using options -tmrca and -subset, and we used bedops (version 2.4.35 (Neph et al. 2012)) to match the times sampled by ARGweaver to the simulated ones at each sequence segment. Finally, we used a custom Python script to calculate the ranks of simulated pairwise coalescence times on ARGweaver MCMC samples per site.

\section{Time discretization}

In ARGweaver, time is discretized such that recombination and coalescence events are only allowed to happen at a user-defined number of time points, $K$ (default value is 20) (Rasmussen et al. 2014). These time points $s_{j}$ (for $0<=j<=K-1$ ) are given by the function

$$
s_{j}=g(j)=\frac{1}{\delta}\left\{e^{\frac{j}{K-1} \log \left(1+\delta s_{K-1}\right)}-1\right\}
$$

where $\delta$ is a parameter determining the degree of clustering of points in recent times. Small values of $\delta$ lead to a distribution of points that is closer to uniform between 0 and $s_{K-1}$, and higher values increase the density of points at recent times (default value is 0.01) (Hubisz and Siepel 2020). Equation 1 ensures that $s_{0}$ is always 0 , and $s_{K-1}$ (or $s_{\max }$ ) is user defined by the parameter-maxtime (default value is 200,000).

Rounding of continuous times into these $K$ time points is done by defining bins with breakpoints between them, such that the breakpoint between times $s_{j}$ and $s_{j+1}$ is $s_{j+\frac{1}{2}}=g\left(j+\frac{1}{2}\right)$. All continuous values in the bin between $s_{j-\frac{1}{2}}$ and $s_{j+\frac{1}{2}}$ are assigned the value $s_{j}$. We note that for the first and last intervals, the values assigned $\left(s_{0}\right.$ and $\left.s_{K-1}\right)$ do not correspond to a midpoint in the time interval but rather to its minimum $\left(s_{0}=0\right)$ or maximum $\left(s_{K-1}=s_{\max }\right)$

Here, when reporting results in bins, we use the same time discretization as defined by the ARGweaver breakpoints $\left(s_{j+\frac{1}{2}}\right)$. However, we change the value assigned to times in these bins: instead of using $s_{j}$, we define $t_{j}$ as the median of the exponential distribution with rate 1 at the interval between $s_{j-\frac{1}{2}}$ 
and $s_{j+\frac{1}{2}}$. To this end, we first calculate the cumulative probability of the exponential distribution with rate 1 up to the median of the $j$ th interval

$$
\begin{aligned}
p_{j} & =\int_{0}^{s_{j-\frac{1}{2}}} e^{-x} \mathrm{~d} x+\frac{1}{2} \int_{s_{j-\frac{1}{2}}}^{s_{j+\frac{1}{2}}} e^{-x} \mathrm{~d} x \\
& =1-\left(\frac{e^{-s_{j-\frac{1}{2}}}+e^{-s_{j+\frac{1}{2}}}}{2}\right)
\end{aligned}
$$

We then take the inverse CDF of the exponential distribution with rate 1 , at the point $p_{j}$, to find the time $t_{j}=-\ln \left(1-p_{j}\right)$ corresponding to the median value for the interval.

This step is relevant for the simulation-based calibration (see below), where we take the rank of true (simulated) coalescence times relative to the values sampled by ARGweaver. If we used $s_{j}$, coalescence times in the first or last ARGweaver time interval would not be represented by a midpoint. We correct for that by using $t_{j}$, so that all time intervals are comparable.

Relate does not use time discretization, and tsdate uses a discretization scheme where the time points are the quantiles of the lognormal prior distribution on node ages (Wohns et al. 2021). Here, we always apply the ARGweaver time discretization scheme when comparing results in bins.

\section{Relate}

VCF files generated with msprime were converted to Relate haps and sample files using RelateFileFormats -mode ConvertFromVcf and Relate's PrepareInputFiles script. We ran Relate (version 1.1.2) using -mode All with the same mutation rate $(-m 2 e-8)$ and effective population size (-N 20000) used in the msprime simulations, as well as a recombination map with constant recombination rate along the genome, with the same rate used in msprime (2e-8).

We used Relate's SampleBranchLengths program to obtain 1000 MCMC samples of coalescence times for the local trees inferred in the previous step in anc/mut output format (-num-samples 1000 -format a). Similarly to the ARGweaver analysis, we also performed this step in 20 sequence segments of $5 \mathrm{Mb}$, and we thinned the results to keep only every 10th MCMC sample. Finally, we extract all pairwise coalescence times and calculate the ranks of true pair- wise coalescence times relative to the $100 \mathrm{MCMC}$ samples.

\section{tsinfer and tsdate}

VCF files generated by msprime were provided as input to the python API using cyvcf2.VCF and converted to tsinfer samples input object using the add_diploid_sites function described in the tsinfer tutorial (https://tsinfer.readthedocs.io/en/latest/tutorial. html\#reading-a-vcf). Genealogies were inferred with tsinfer (version 0.2.0 (Kelleher et al. 2019)) with default settings and dated with tsdate (version 0.1.3 (Wohns et al. 2021)) using the same parameter values as in the simulations $(\mathrm{Ne}=10000$, mutation_rate $=2 e-8)$, with a prior grid of 20 timepoints.

Pairwise coalescence times were extracted from the tree sequences using functions get_interval() and tmrca() from tskit (version 0.3.4 (Kelleher et al. 2018)), and coalescence times at each site were matched to the simulated ones using bedops (Neph et al. 2012).

\section{MCMC convergence}

We evaluated MCMC convergence of Relate and ARGweaver through 1) visual inspection of trace plots, 2) autocorrelation plots, 3) effective sample sizes and 4) the Gelman-Rubin convergence diagnostics based on potential scale reduction factor (Gelman and Rubin 1992; Brooks and Gelman 1998). Trace plots were also used to determine the number of burn-in samples, and autocorrelation plots were used to determine thinning of the samples. See Evaluating MCMC Convergence in Supplementary Materials for details.

\section{Point estimates of pairwise coalescence times}

We estimated pairwise coalescence times from the MCMC samples from Relate and ARGweaver by taking the average of 100 samples at each site. Since tsdate does not output multiple samples of node times, we use its point estimate of pairwise coalescence times directly.

Mean squared error (MSE) of point estimates was calculated per bin of size 0.1 of the simulated coalescence times (in units of $2 N_{e}$ generations). In each bin, we calculated the square of the difference between point estimates and simulated coalescence times at each sequence tract (i.e., the squared error) and then calculated an average of those values weighted by length of the sequence tracts. MSE per simulation was calculated as the weighted average 
of the squared errors in each bin of size 0.1 , where the weights were proportional to the number of sites in each bin.

\section{Simulation-based calibration}

In addition to comparing MCMC point estimates to the true simulated values, we use simulation methods proposed by Cook et al. (2006) and Talts et al. (2020) to assess whether Bayesian methods are sampling correctly from the true posterior distribution. Cook et al. (2006) proposed simulating data using parameters sampled from the prior. The posterior, when averaged over multiple simulated data sets, should then equal the prior.

In our case, we sample ARGs, $G$, from the full coalescence process with recombination with a known implicit prior of pairwise coalescence times, $P(t)=$ $e^{-t}$. We simultaneously simulate sequence data, $x$, on the simulated ARGs from the distribution $p(x)=\int p(x \mid G) \mathrm{d} P(G)$. The distribution of the averaged posterior of $G, p_{\text {ave }}(G)=\int p(G \mid X) \mathrm{d} P(x)$ should then equal the prior for $G$ (Talts et al. 2020), and hence the prior distribution for the pairwise coalescence times, $t$, should equal the averaged posterior distribution for $t$. Here, all population parameters relating to mutation, effective population sizes, etc., are kept fixed and suppressed in the notation. One way we will examine the accuracy of the posterior inferences is, therefore, to compare the average of the posterior of $t$ to the exponential distribution. In practice, we simulate data using msprime (Kelleher et al. 2016) and pipe the data to the MCMC samplers (ARGweaver and Relate) for inference of the posterior distribution. ARGweaver uses an approximation (SMC') of the model (coalescent with recombination) used in the data simulations, and Relate uses a heuristic method based on the Li and Stephens model. Thus, inadequacies of the fit of the posteriors could potentially be caused by this discrepancy between the model used in simulations and the models used for inference.

However, even if the averaged posterior resembles an exponential, the inferences for any particular value of $t$ may have a posterior that is too narrow or too broad. For a closer examination of the accuracy of the posterior, we use a method proposed by Cook et al. (2006) and Talts et al. (2020) that compares each posterior to the true value. To this end, we compare each true (simulated) pairwise coalescence time to the corresponding posterior for the same pair of hap- lotypes. If the posterior is correctly calculated, the rank of the true value relative to the samples from the posterior should be uniformly distributed (Cook et al. (2006), Talts et al. (2020)). We use $100 \mathrm{MCMC}$ samples from ARGweaver and Relate for each data set, meaning our ranks take values from 0 to 100 . Deviations from the uniform distribution of ranks quantifies inaccuracies in estimation of the posterior. For example, an excess of low and high ranks indicates that the inferred posterior distribution is underdispersed relative to the true posterior.

The authors affirm that all data necessary for confirming the conclusions of the article are present within the article, figures, and tables.

\section{Results}

\section{Comparison of simulated to estimated coalescence time per site}

We compared coalescence times estimated by ARGweaver, Relate and tsdate to the true values known from msprime simulations. In all three methods, estimates of coalescence time per site are biased (Figure 2 and S2). Small values of coalescence times are generally overestimated, while large values tend to be underestimated (Fig S2). In tsdate, point estimates are apparently bounded to a narrow range (Figure 2G). The mean squared error (MSE) of point estimates is larger in Relate (MSE=0.625) and tsdate (MSE=0.806) than in ARGweaver (MSE=0.397), showing that point estimates of pairwise coalescence times at each site are closer to the true value in ARGweaver.

For ARGweaver and Relate, the point estimates of coalescence times are obtained as the means of samples from the posterior. These Bayesian estimates are not designed to be unbiased and unbiasedness of the point estimator is arguably not an appropriate measure of performance for a Bayesian estimator. Therefore, we also evaluate the degree to which the posterior distributions reported by ARGweaver and Relate are well calibrated, i.e. represent distributions that can be interpreted as valid posteriors, and the degree to which the data-averaged posterior distributions of coalescence times equals the prior exponential distribution.

\section{Posterior distribution of coalescence times}

We simulated data under the standard coalescent model, where the distribution of pairwise coalescence times (in units of $2 N_{e}$ generations, where $N_{e}$ is 
the diploid effective population size) follows an exponential distribution with rate parameter 1 (Figure S3). As argued in the Methods section, the same is true for the data-averaged posterior.

We compared the expected exponential distribution of coalescence times to the observed distribution of coalescence times across all sites inferred by ARGweaver, Relate, and tsdate (Figure 3). For ARGweaver and Relate, we output 100 MCMC samples from the posterior distribution and plot the distribution of pairwise coalescence times across all sites and MCMC samples.

To facilitate visual comparison of the distributions between methods, we discretized Relate and tsdate coalescence times into the same bins as ARGweaver (Figure 3D,G, see distributions without discretization in Figure S4 and see Methods for a description of ARGweaver time discretization). Because the time discretization breakpoints are regularly spaced on a $\log$ scale, we use a log scale on the $\mathrm{x}$-axis for better visualization.

Distributions of coalescence times from ARGweaver and Relate (Figure 3A and D) show an excess around 1 , when compared to the expected exponential distribution. However, that bias is more pronounced in Relate than ARGweaver. In tsdate, the distribution is truncated at 1.6, and it deviates more strongly from the expected exponential distribution (Figure 3G). We note that the plots from ARGweaver and Relate are not directly comparable to those of tsdate, since there are 100 coalescence time samples at each site from the former two programs but only one from tsdate.

\section{Simulation-based calibration}

In this section, we use simulation-based calibration to evaluate whether ARGweaver and Relate are generating samples from a valid posterior distribution of coalescence times (see Methods). To that end, we simulated coalescence times at multiple sites following the standard coalescent prior distribution, and we generated $100 \mathrm{MCMC}$ samples from the posterior distribution using both ARGweaver and Relate. Finally, we analyse the distribution of the ranks of the simulated coalescence times relative to the 100 sampled values at each site.

In the previous section, we showed that the posterior distributions of ARGweaver and Relate are similar to the theoretically expected exponential distribution. However, in that analysis we have not evaluated the distribution of MCMC samples relative to each simulated value. The results of simulationbased calibration are informative about that distribution and can reveal if the posterior distribution is well calibrated.

The distribution of ranks from ARGweaver (Figure 4A, Kullback-Leibler Divergence $(K L D)=0.027$ ) is closer to uniform than that of Relate (Figure 4D, $\mathrm{KLD}=0.602$ ). However, both show an excess of low and high ranks. The excess of low and high ranks indicates that the sampled posterior distribution is underdispersed (Talts et al. 2020), i.e. the posterior has too little variance and does not represent enough uncertainty regarding the coalescence times.

One possible cause for this type of deviation from the uniform distribution could be MCMC convergence, i.e., samples being autocorrelated, resulting in effective sample size is lower than the number of samples taken, the MCMC chain not mixing well and/or the MCMC chain not being run long enough to achieve convergence.

We show detailed results for MCMC convergence in Relate and ARGweaver in the Supplementary Materials. Briefly, we have not found these types of convergence issues in ARGweaver or Relate with simulations of 8 haplotypes and mutation to recombination ratio of 1 . Potential scale reduction factor (PSRF) from Gelman-Rubin convergence diagnostic statistics are all close to 1 (Tables S2, S3), and effective sample sizes are almost all larger than 100. Therefore, MCMC convergence does not seem to explain why the rank distributions are not uniform.

\section{Increased mutation to recombination ratio}

When inferring an ARG from sequence data, the information for inference comes from mutations that cause variable sites in the sequence data. The lower the recombination rate, the longer the span of local trees will be and the more mutations will be available to provide information about each local tree. More generally, an increased mutation to recombination ratio is expected to increase the amount of information available to infer the ARG.

In our standard simulations presented so far, the mutation to recombination ratio is one $(\mu=\rho=$ $2 * 10^{-8}$ ). We increased the simulated mutation to recombination ratio to 10 , both by decreasing the recombination rate $(\rho)$ tenfold and also by increasing the mutation rate $(\mu)$ tenfold. We expected that these scenarios would improve inference of ARGs. Point 
estimates are better with increased mutation to recombination ratio in ARGweaver (Figure 2B,C) and Relate (Figure 2E,F). However, the same is not true of tsdate, where the mean squared error for point estimates decreases with lower recombination rate but increases with higher mutation rate in comparison to the scenario of equal mutation and recombination rates (Figure 2H,I). On the other hand, the posterior distribution of coalescence times in tsdate improved with increasing mutation rate (Figure 3I).

The coalescence times distribution in Relate (Figure $3 \mathrm{E}, \mathrm{F})$ are closer to the expected with $\mu / \rho=10$ relative to $\mu / \rho=1$ (Fig 3D), and the simulation-based calibration also improved (Figure 4D-F, KLD=0.492 and 0.498 compared to KLD $=0.602$ ).

The results from ARGweaver with $\mu / \rho=10$ were more surprising, with the simulation-based calibration showing a more pronounced underdispersion of the posterior distribution (Figure $4 B, C, K L D=0.286$ and 0.350 , compared with $\mathrm{KLD}=0.027$ for $\mu / \rho=1$ ). The overall distribution of coalescence times, however, showed little change (Figure 3B,C). One possible explanation for ARGweaver results being worse with higher mutation to recombination ratio might be that MCMC mixing is worse under those conditions, leading to convergence issues not observed for the previous scenario. Examining convergence diagnostics seems to confirm this with more coalescence times showing low effective sample size, and with a potential scale reduction factor showing evidence of lack of convergence of some coalescence times (see Evaluating MCMC Convergence in Supplementary Materials).

We show additional results of ARGweaver on simulations with intermediate values of $\mu / \rho$ (2 and 4), under the SMC and $\mathrm{SMC}^{\prime}$ genealogy models, and with the Jukes-Cantor mutation model in the Supplementary Materials.

\section{Number of samples}

Next, we evaluate ARG inference with simulations with different sample sizes. Our standard sample size used so far was 8 haplotypes, and here we change it to 4,16 and 32. For Relate and tsdate, which are scalable to larger sample sizes, we also evaluated inference with 80 and 200 sampled haplotypes.

For ARGweaver, increasing sample sizes decreased the MSE of point estimates (Figure 5A-C), distributions of coalescence times remained similar (Figure 6A-C), but underdispersion of the posterior distribution increased (Figure 7A-C). As mentioned in the previous section, this could be caused by an MCMC mixing problem. In particular, a larger number of samples will contribute to an increasing number of states for ARGweaver to explore, possibly leading to poor MCMC convergence (see Evaluating MCMC Convergence).

With a smaller sample size ( $\mathrm{n}=4$ haplotypes), the coalescence time distribution from Relate showed an excess around the mean value (coalescence time of 1) (Fig 6D). With increasing sample sizes, it became more similar to the expected distribution (Fig 6E-H). Calibration of the posterior distribution improved with increasing sample sizes up to 32 haplotypes (Figure 7D-H).

Both the point estimates and posterior distribution of coalescence times in tsdate do not consistently improve or worsen with increasing sample sizes in the range tested here (Figures 5I-M and 6I-M).

\section{Runtime}

We point out that runtimes differ widely among the programs compared here, and this factor should be taken into account for users making decisions on what method to use for their applications. For example, in the simulations with mutation rate equal to recombination rate, with sample size of 8 haplotypes and taking 1000 MCMC samples, ARGweaver took a total of 641 computing hours while Relate took 17 hours. The clock time was reduced by running both programs in parallel for segments of $5 \mathrm{Mb}$ of the total $100 \mathrm{Mb}$ sequence, meaning that ARGweaver took approximately $35 \mathrm{~h}$. However, this still could be a significant amount of time for the user, depending on their utilization of the algorithm. For a systematic comparison of runtimes between Relate and ARGweaver, see Speidel et al. (2019). tsinfer and tsdate took only 5 minutes, although that time is not comparable to ARGweaver and Relate because it does not include the time consuming step of estimating a posterior using MCMC.

\section{Discussion}

ARG inference promises to be a tremendously useful tool for inferences of evolutionary history, such as natural selection or demography. However, it is also a very hard computational problem. We compared methods that use different approaches to this problem and evaluated their accuracy using simulated 

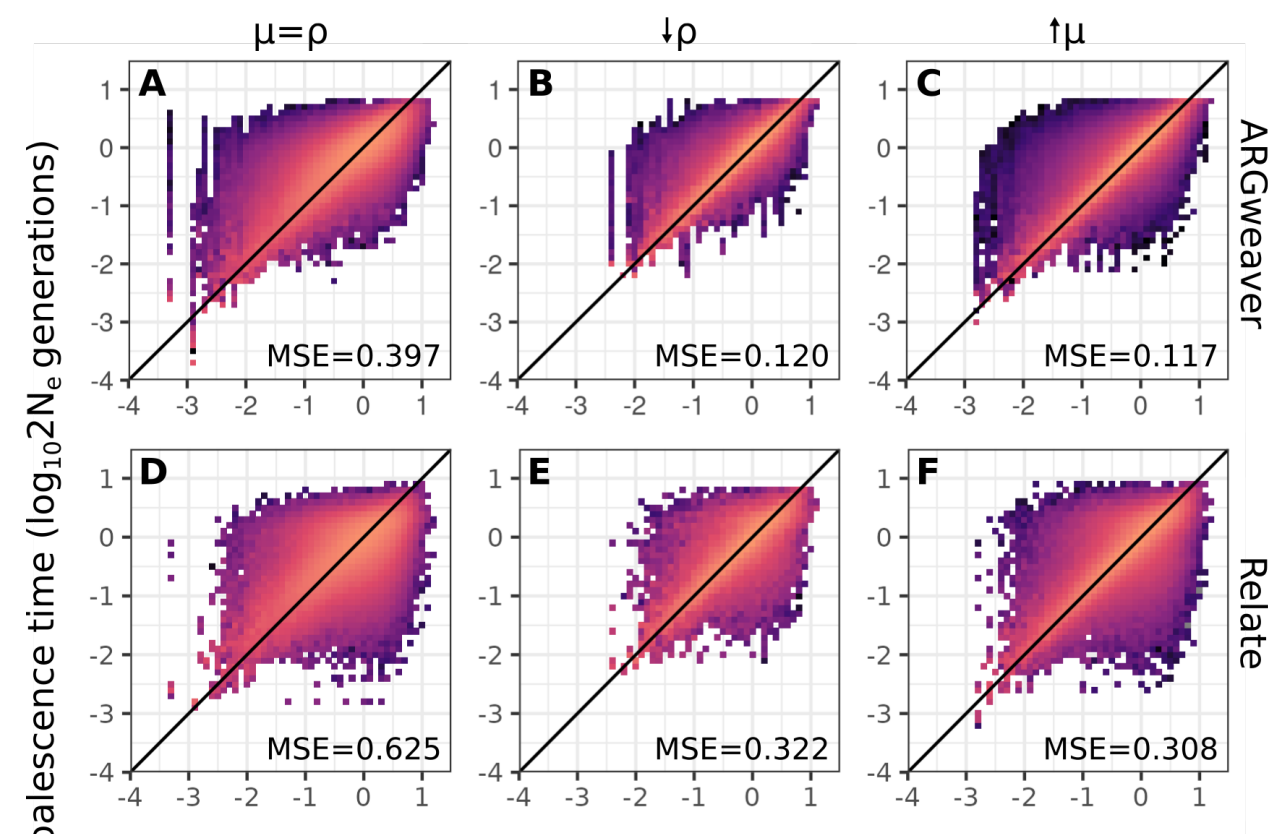

nSites
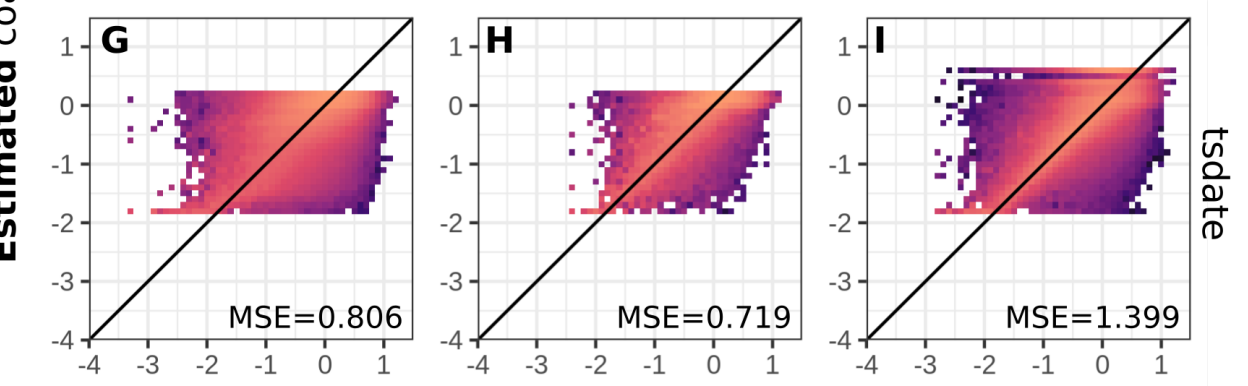

Simulated coalescence time ( $\log _{10} 2 \mathrm{~N}_{\mathrm{e}}$ generations)

Figure 2 Point estimates of coalescence times in ARGweaver (A-C), Relate (D-F) and tsdate (G-I). Left column: $\mu=\rho=2 \times 10^{-8}$; middle column: $\mu / \rho=10, \rho=2 \times 10^{-9}$; right column: $\mu / \rho=10, \mu=2 \times 10^{-7}$. For ARGweaver and Relate, point estimates are the means of $100 \mathrm{MCMC}$ iterations. Note that axes are in log scale. See Figure S1 for the data in plots A,D,G plotted in linear axes. Diagonal line shows x=y. 

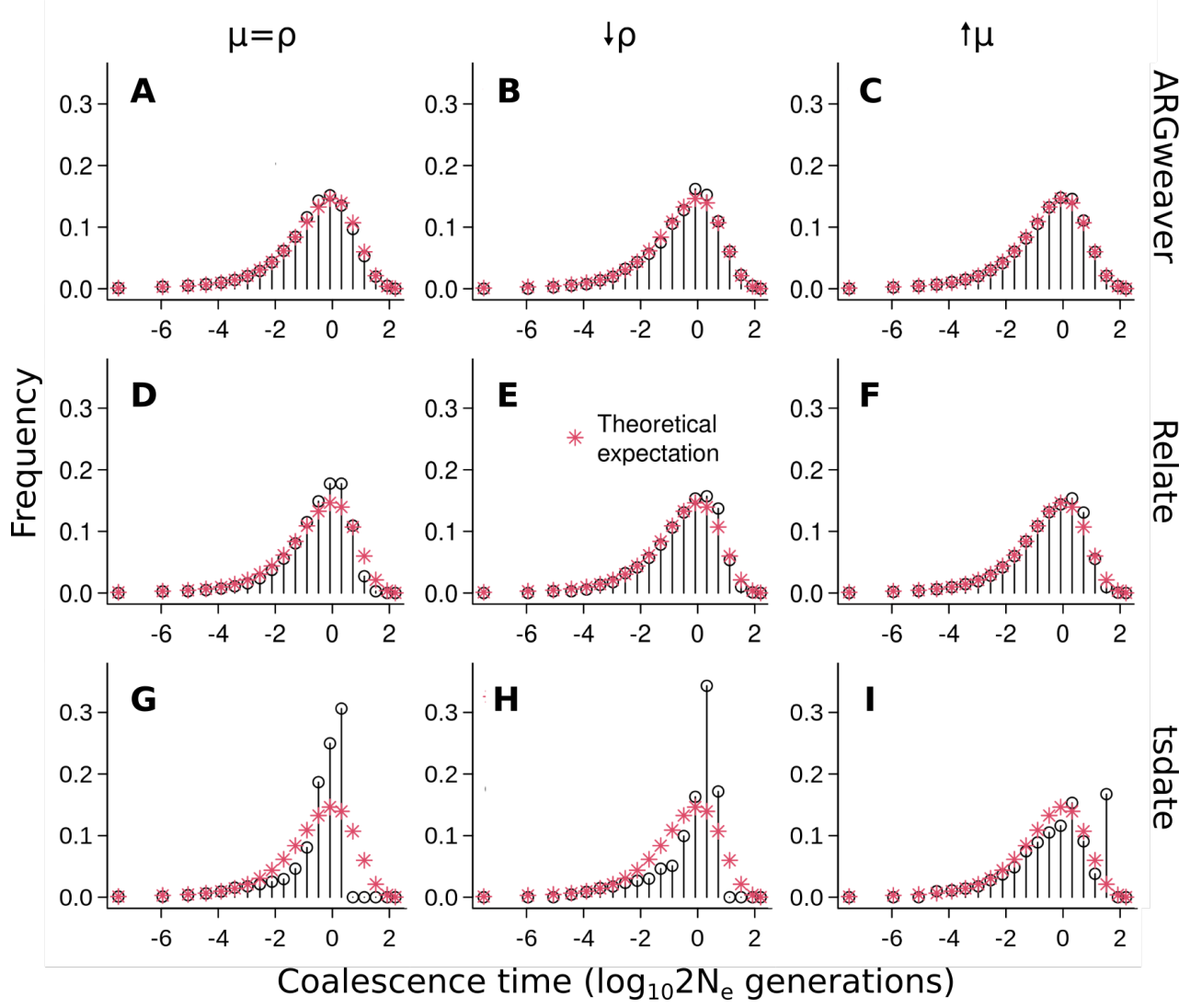

Figure 3 Distribution of coalescence times inferred by ARGweaver (A-C), Relate (D-F) and tsdate (G-I). Left column: $\mu=\rho=2 * 10^{-8}$; middle column: $\mu / \rho=10, \rho=2 * 10^{-9}$; right column: $\mu / \rho=10, \mu=2 * 10^{-7}$. Plots $\mathrm{D}$ and $\mathrm{G}$ show the same data as in Figure $\mathrm{S} 4$, using different binning. 


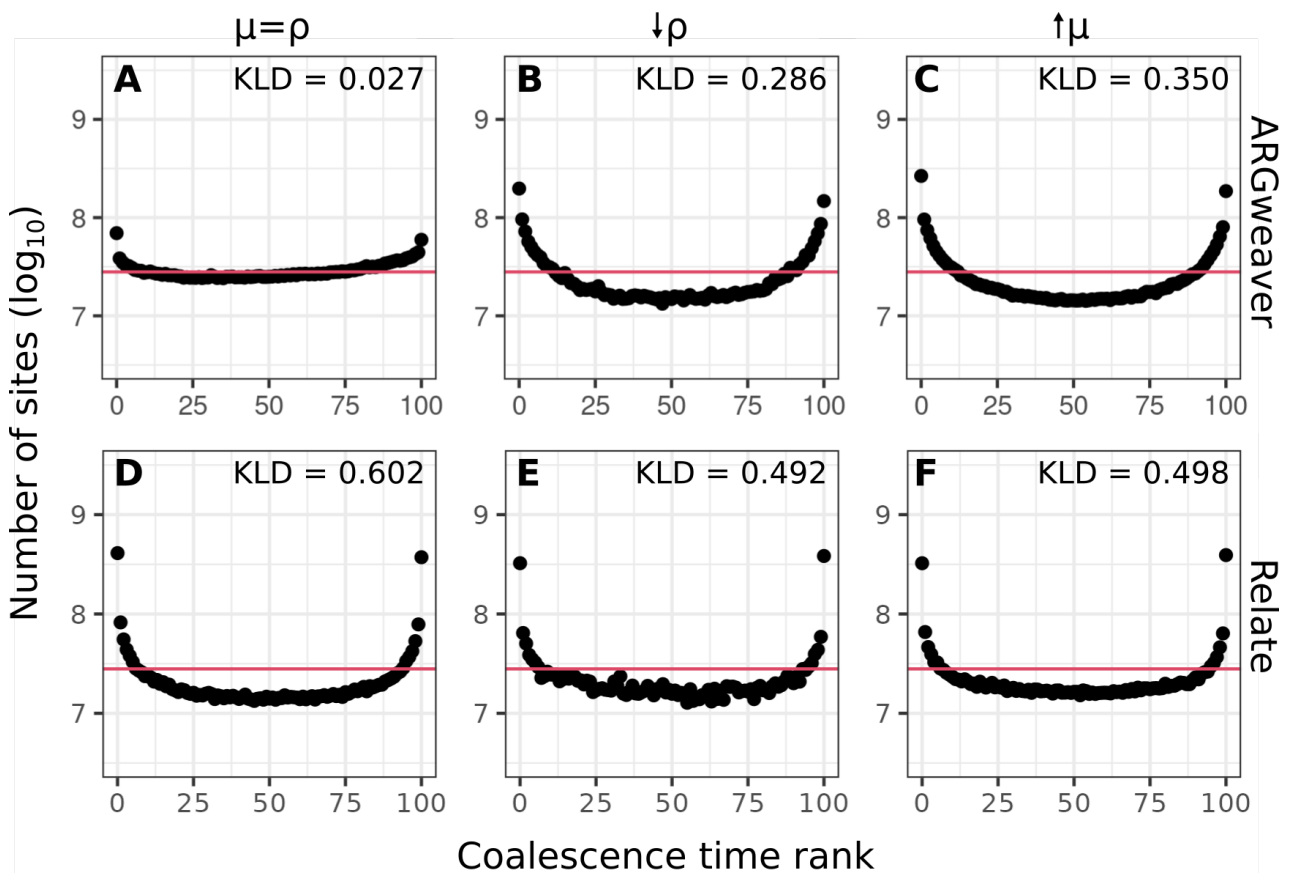

Figure 4 Counts of ranks from simulation-based calibration in ARGweaver (a-c) and Relate (d-f). Horizontal line shows expected uniform distribution. Left column: $\mu=\rho=2 * 10^{-8}$; middle column: $\mu / \rho=10$ decreasing recombination rate $\left(\rho=2 * 10^{-9}\right)$; right column: $\mu / \rho=10$ increasing mutation rate $\left(\mu=2 * 10^{-7}\right)$. Horizontal line shows expected uniform distribution.

to a more centered, under-dispersed distribution, as shown by the larger deviations from the uniform distribution in simulation-based calibration (Figures 4 and 7, where ARGweaver KLD values range from 0.027 to 0.313 , and Relate range from 0.429 to 0.938 ).

Additionally, we showed that Relate's inferences generally improve with sample size (Figures 5, 6, 7). This is expected from inference using the $\mathrm{Li}$ and Stephens (2003) copy algorithm, which tends to better approximate the genealogical process with larger samples sizes. Because Relate is fast enough, even for thousands of samples, it is preferred for large numbers of genomes - not only because ARGweaver is not scalable for such large sample sizes but also because Relate inference tends to improve with larger sample sizes (Hubisz and Siepel 2020).

The framework of tsinfer and tsdate is also based on the Li and Stephens (2003) model, and it additionally takes advantage of the succinct tree sequence data structure that makes it scalable to even larger sample sizes than Relate, and at least an order of magnitude larger than tested here (Wohns et al. 2021). Although we did not find an improvement of tsdate estimates with increasing sample sizes in the range we tested (4 to 200 haplotypes), our analyses cannot 

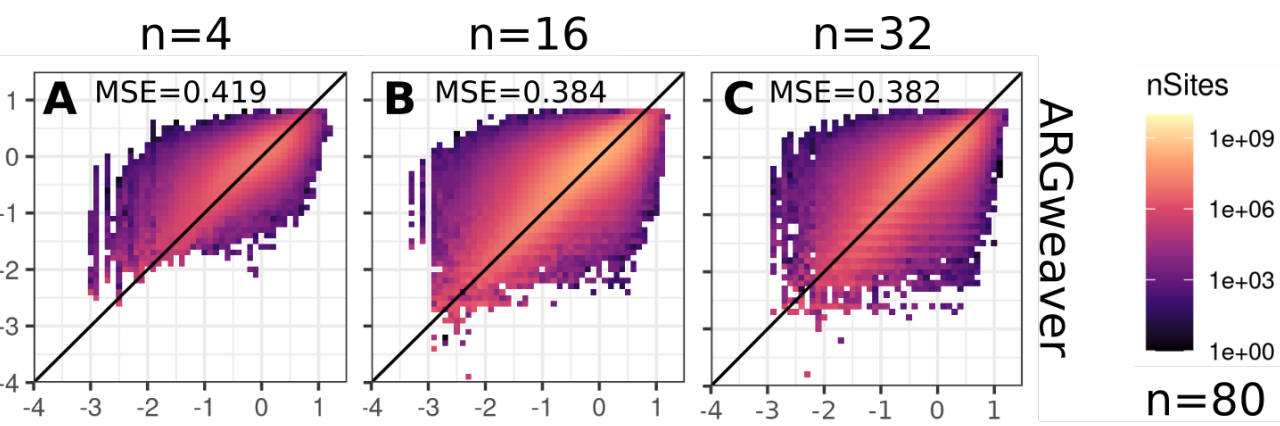

nSites

$1-D \mathrm{MSE}=0.676$
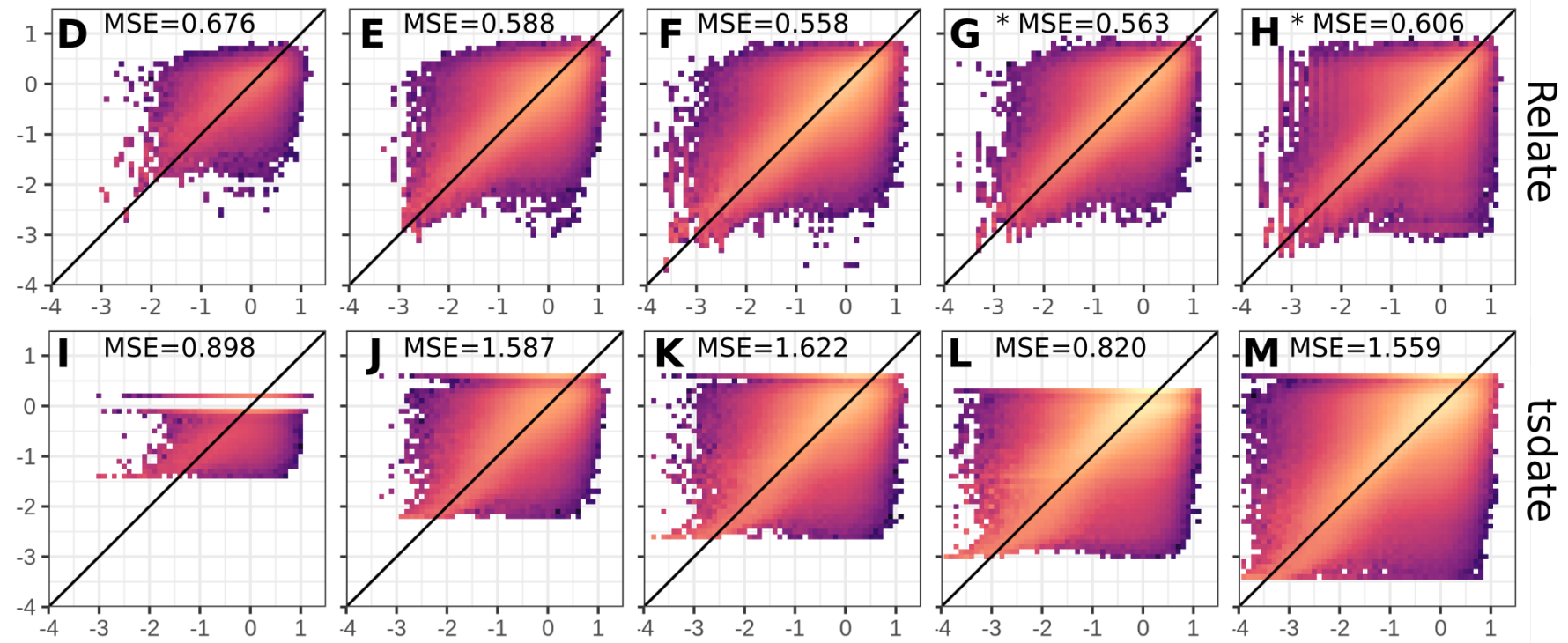

\section{Simulated coalescence time ( $\log _{10} 2 \mathrm{~N}_{\mathrm{e}}$ generations)}

Figure 5 Point estimates of ARGweaver (A-C), Relate (D-H) and tsdate (I-M). Columns show different number of simulated samples 4, 16, 32, 80 or 200 haplotypes. Mean squared error (MSE) is shown for each plot. Note that ARGweaver is not scalable for simulations with larger sample sizes. * indicate results for a subset of 210 pairs of samples, instead of all pairwise coalescence times. 

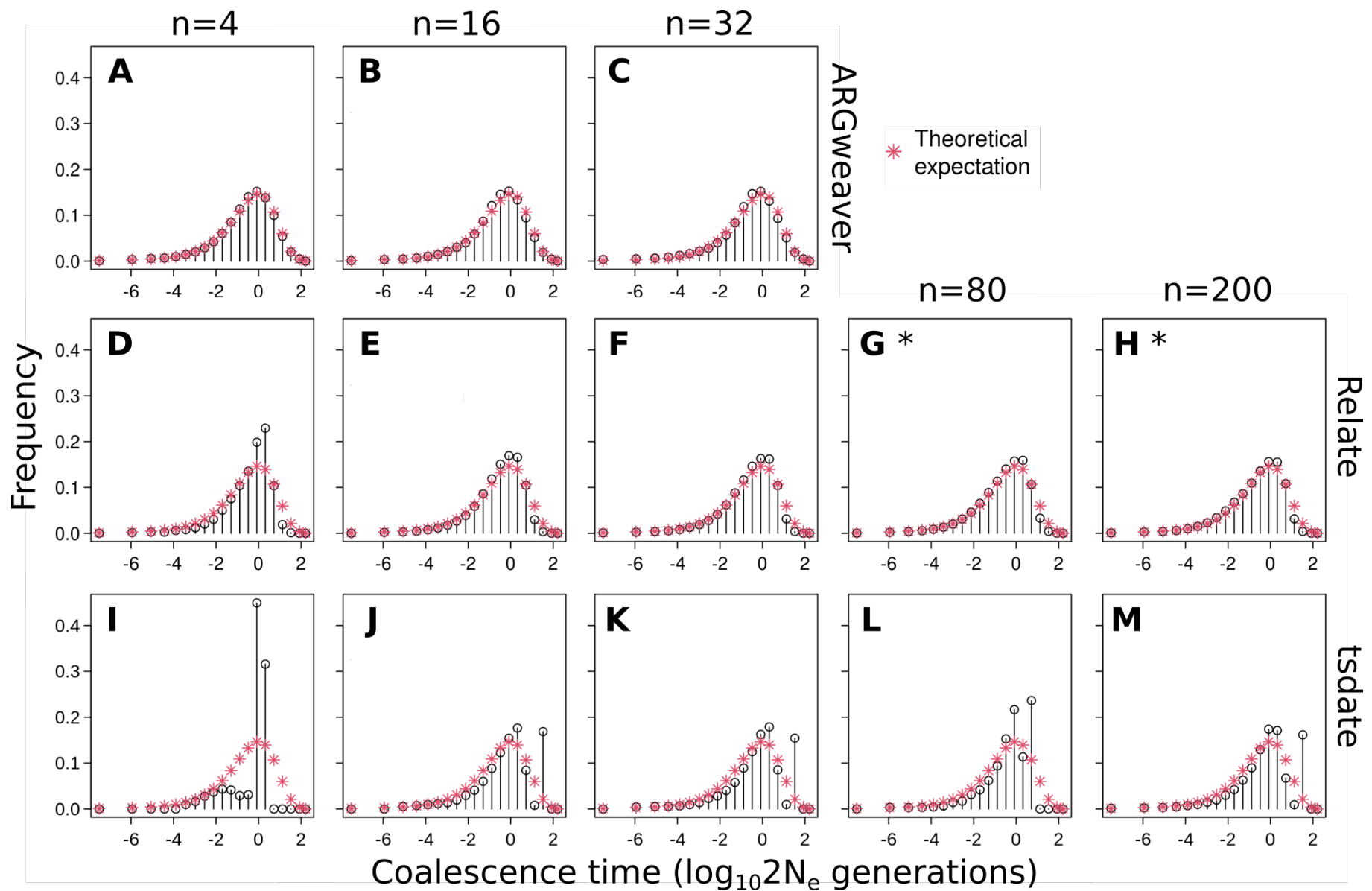

Figure 6 Distribution of coalescence times in ARGweaver (A-C), Relate (D-H) and tsdate (I-M). Columns: sample sizes of 4, 16, 32, 80, 200 haplotypes. * indicate results for a subset of 210 pairs of samples, instead of all pairwise coalescence times. 


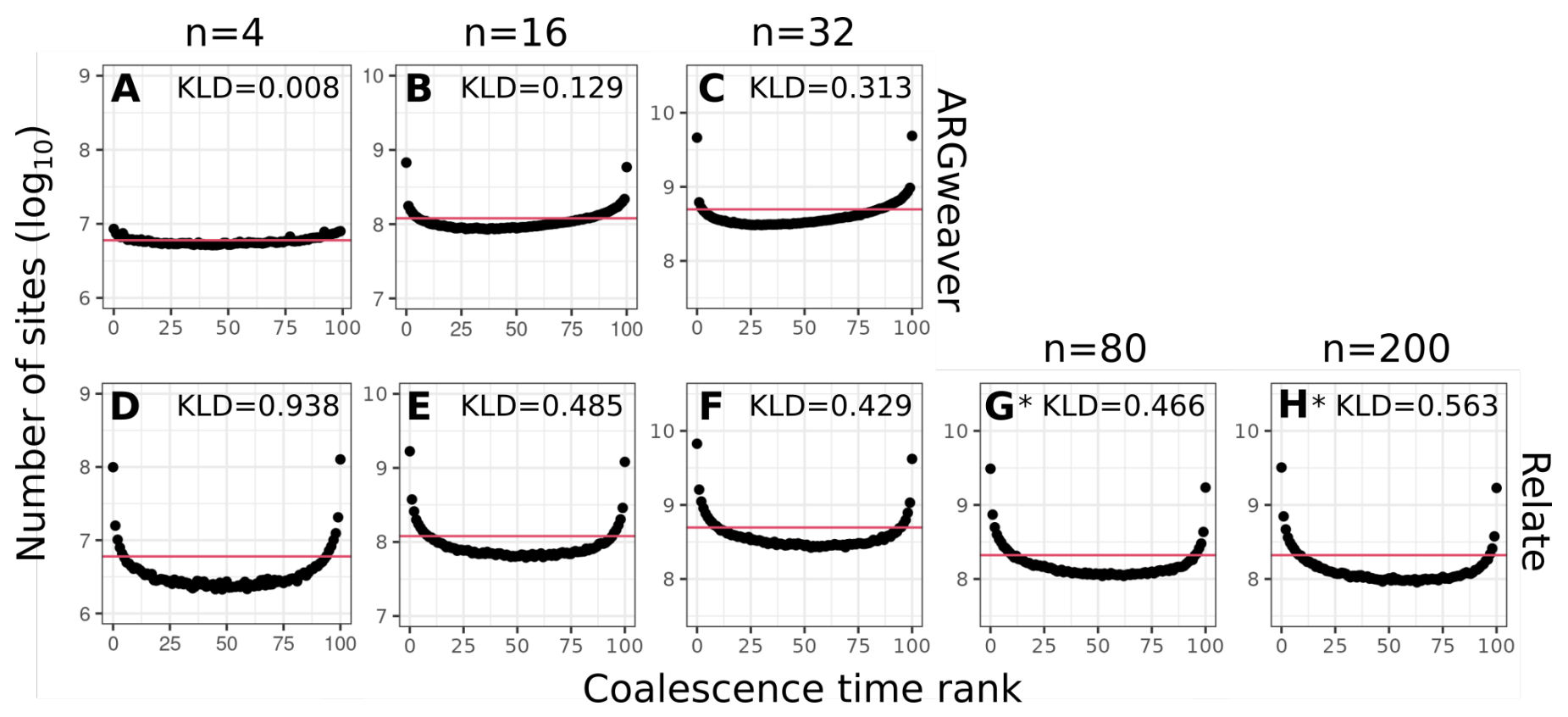

Figure 7 Simulation-based calibration for ARGweaver (A-C) and Relate (D-H). Columns: sample sizes of $4,16,32,80,200$ haplotypes. Horizontal line shows expected uniform distribution. Note that the y-axis is centralized on different values but always has the same length. * indicate results for a subset of 210 pairs of samples, instead of all pairwise coalescence times.

rule out the possibility of better tsdate inference at larger sample sizes.

Increasing the mutation to recombination ratio in simulations improved point estimates from ARGweaver but did not improve posterior calibration (Figure 4). This lack of improvement of the posterior sampling can be explained by lack of convergence and could potentially be improved by increasing the number of MCMC iterations. Although the statistics recorded by ARGweaver at each iteration (likelihood, number of recombinations, etc.) show convergence (Figure S5, Table S1), we observed that certain pairwise coalescence times did not converge in the simulations with increased mutation to recombination ratio (Table S2 see more discussion in ARGweaver in Supplementary Materials).

\section{Other features of methods not explored in our sim- ulations}

Although the focus of this study is coalescence time, some other features could also be relevant for applications to real data. For example, sequencing or phasing errors could reduce the performance. ARGweaver is the only method compared here that supports unphased data. In addition, it can take into account local variation in coverage, mapping quality, sequencing errors, and incorporate genotype probabilities, all of which are features not tested here. ARGweaver can also incorporate a map of variable mutation rates. Both ARGweaver and Relate can incorporate maps of variable recombination rates across the genome, features which were not used in our constant rate simulations.

In our standard simulations, we use mutation rate equal to recombination rate, which is believed to be approximately true for humans. In reality, even if average recombination and mutation rates are similar, the average recombination rate is not distributed equally along the genome in humans and other mammals but is concentrated in recombination hotspots. Therefore, it is possible that ARG inference could be more accurate with real data, since local trees could span longer sequences separated by recombination hotspots.

\section{Acknowledgements}

We would like to thank Leo Speidel for identifying an error in the extraction of coalescence times from the Relate output in a previous version of the manuscript. 


\section{Funding}

This material is based upon work supported by NSF Graduate Research Fellowship No. 2146752 awarded to Andrew Vaughn and by NIH grant R01GM138634 awarded to Rasmus Nielsen.

\section{Literature Cited}

Baumdicker, F., G. Bisschop, D. Goldstein, G. Gower, A. P. Ragsdale, et al., 2021 Efficient ancestry and mutation simulation with msprime 1.0. bioRxiv 17: 2021.08.31.457499.

Brooks, S. P. and A. Gelman, 1998 General methods for monitoring convergence of iterative simulations. Journal of Computational and Graphical Statistics 7: 434-455.

Cook, S. R., A. Gelman, and D. B. Rubin, 2006 Validation of software for Bayesian models using posterior quantiles. Journal of Computational and Graphical Statistics 15: 675-692.

Deng, Y., Y. S. Song, and R. Nielsen, 2021 The distribution of waiting distances in ancestral recombination graphs. Theoretical Population Biology

Gelman, A. and D. B. Rubin, 1992 Inference from iterative simulation using multiple sequences. Statistical Science 7: 457-472.

Griffiths, R. C. and P. Marjoram, 1997 An Ancestral Recombination Graph. In Progress in Population Genetics and Human Evolution, IMA Volumes in Mathematics and its Applications, vol. 87, edited by P. Donnelly and S. Tavare, pp. 257-270, Springer.

Hubisz, M. and A. Siepel, 2020 Inference of Ancestral Recombination Graphs Using ARGweaver. In Statistical Population Genomics, edited by Julien Y. Dutheil, volume 2090, chapter 10, pp. 231-266.

Hubisz, M. J., A. L. Williams, and A. Siepel, 2020 Mapping gene flow between ancient hominins through demography-aware inference of the ancestral recombination graph. PLoS genetics 16: e1008895.

Hudson, R. R., 1983 Properties of a neutral allele model with intragenic recombination. Theoretical Population Biology 23: 183-201.

Jukes, T. H. and C. R. Cantor, 1969 Evolution of Protein Molecules. In Mammalian Protein Metabolism, edited by H. N. Munro, chapter 24, pp. 21-132, Academic Press.

Kelleher, J., A. M. Etheridge, and G. McVean, 2016 Efficient Coalescent Simulation and Genealogical
Analysis for Large Sample Sizes. PLoS Computational Biology 12: 1-22.

Kelleher, J., K. R. Thornton, J. Ashander, and P. L. Ralph, 2018 Efficient pedigree recording for fast population genetics simulation. PLOS Computational Biology 14: 1-21.

Kelleher, J., Y. Wong, A. W. Wohns, C. Fadil, P. K. Albers, et al., 2019 Inferring whole-genome histories in large population datasets. Nature Genetics 51: 1330-1338.

Kingman, J. F. C., 1982 On the Genealogy of Large Populations. Technical report.

Li, N. and M. Stephens, 2003 Modelling Linkage Disequilibrium using Single Nucleotide Polymorphism Data 2233: 2213-2233.

Marjoram, P. and J. D. Wall, 2006 Fast "coalescent" simulation. BMC Genetics 7: 16.

McVean, G. A. T. and N. J. Cardin, 2005 Approximating the coalescent with recombination. Philosophical transactions of the Royal Society of London. Series B, Biological sciences 360: 1387-93.

Neph, S., M. S. Kuehn, A. P. Reynolds, E. Haugen, R. E. Thurman, et al., 2012 BEDOPS: highperformance genomic feature operations. Bioinformatics 28: 1919-1920.

Osmond, M. and G. Coop, 2021 Estimating dispersal rates and locating genetic ancestors with genomewide genealogies. bioRxiv p. 2021.07.13.452277.

Plummer, M., N. Best, K. Cowles, and K. Vines, 2006 CODA: Convergence Diagnosis and Output Analysis for MCMC. R News 6: 7-11.

Ralph, P., K. Thornton, and J. Kelleher, 2020 Efficiently Summarizing Relationships in Large Samples: A General Duality Between Statistics of Genealogies and Genomes. Genetics 215: 779 LP 797.

Rasmussen, M. D., M. J. Hubisz, I. Gronau, and A. Siepel, 2014 Genome-Wide Inference of Ancestral Recombination Graphs. PLoS Genetics 10.

Roy, V., 2020 Convergence diagnostics for Markov Chain Monte Carlo.

Sheehan, S., K. Harris, and Y. S. Song, 2013 Estimating variable effective population sizes from multiple genomes: a sequentially Markov conditional sampling distribution approach. Genetics 194: 647662.

Speidel, L., M. Forest, S. Shi, and S. R. Myers, 2019 A method for genome-wide genealogy estimation for thousands of samples. Nature Genetics 51: 13211329. 
Steinrücken, M., J. Kamm, J. P. Spence, and Y. S. Song, 2019 Inference of complex population histories using whole-genome sequences from multiple populations. Proceedings of the National Academy of Sciences 116: 17115-17120.

Steinrücken, M., J. P. Spence, J. A. Kamm, E. Wieczorek, and Y. S. Song, 2018 Model-based detection and analysis of introgressed Neanderthal ancestry in modern humans. Molecular ecology 27: 38733888.

Stephens, M. and P. Donnelly, 2000 Inference in molecular population genetics. Journal of the Royal Statistical Society: Series B (Statistical Methodology) 62: 605-635.

Stern, A. J., P. R. Wilton, and R. Nielsen, 2019 An approximate full-likelihood method for inferring selection and allele frequency trajectories from DNA sequence data. PLoS Genetics 15: 1-32.

Taboga, M., 2017 Markov Chain Monte Carlo (MCMC) diagnostics. Kindle Direct Publishing, third edition. Talts, S., M. Betancourt, D. Simpson, A. Vehtari, and A. Gelman, 2020 Validating Bayesian Inference Algorithms with Simulation-Based Calibration. arXiv pp. 1-19.

Wilton, P. R., S. Carmi, and A. Hobolth, 2015 The $\mathrm{SMC}^{\prime}$ is a highly accurate approximation to the ancestral recombination graph. Genetics 200: 343355.

Wiuf, C. and J. Hein, 1999 Recombination as a point process along sequences. Theoretical Population Biology 55: 248-259.

Wohns, A. W., Y. Wong, B. Jeffery, A. Akbari, S. Mallick, et al., 2021 A unified genealogy of modern and ancient genomes. bioRxiv . 


\section{Supplementary Materials}

\section{Figures}

(a)

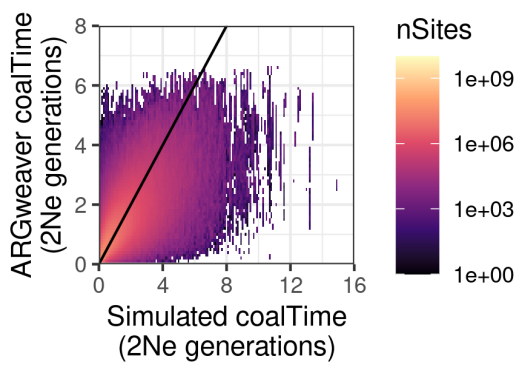

(b)

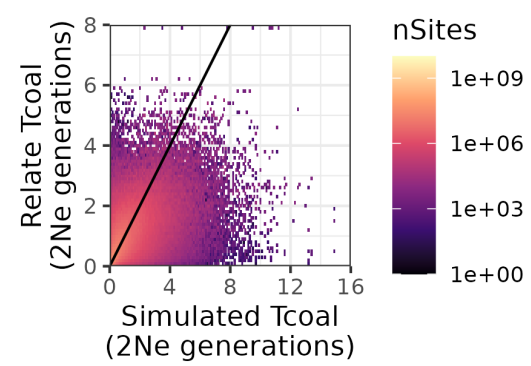

(c)

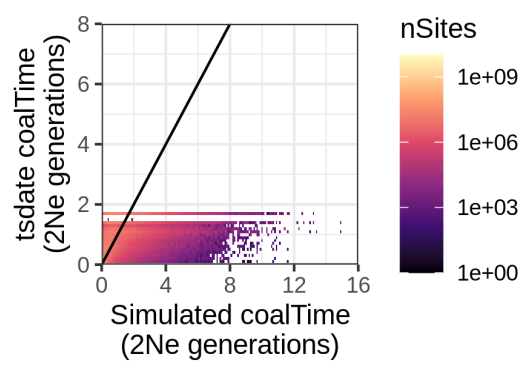

Figure S1 True pairwise coalescence time from msprime simulations compared to inferred coalescence time from (A) ARGweaver (B) Relate (C) tsdate. Note that axes are in linear scale. See Figure 2A, D, G for this data plotted on a logarithmic scale. These results are for simulations with $\mathrm{n}=8$ samples (haplotypes), mutation and recombination rates of $2 \times 10^{-8}$. Diagonal line shows $x=y$, points show the mean inferred coalescence time within a true coalescence time bin. 
(a)

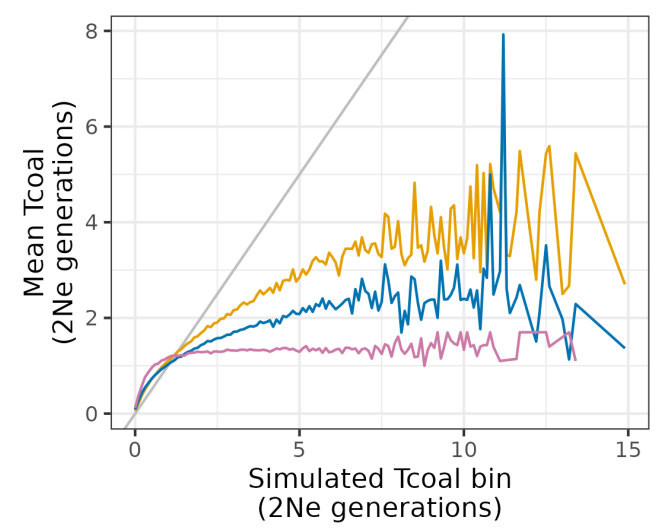

(c)

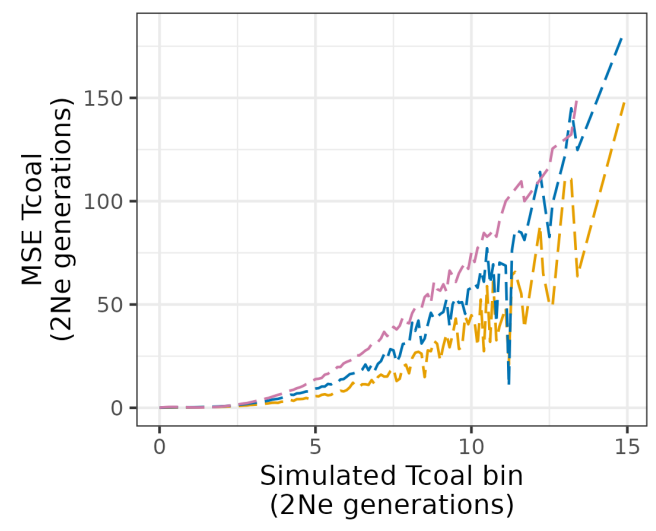

(b)

Program

- ARGweaver

- Relate

- tsdate

Program

- - ARGweaver

- - Relate

- - tsdate

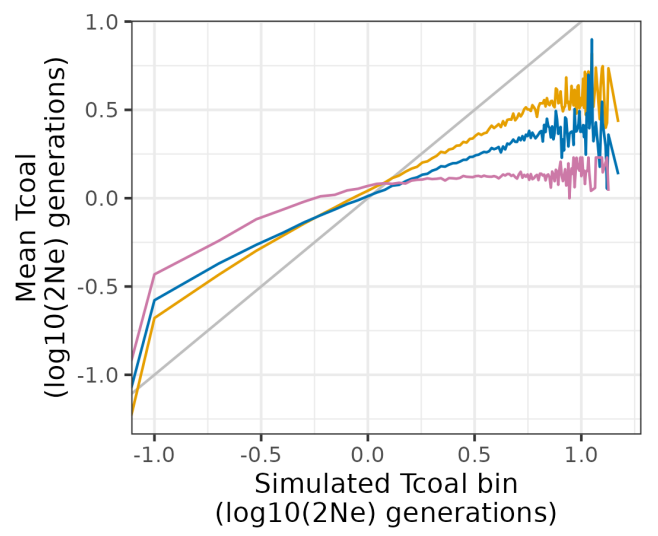

Program

- ARGweaver

- Relate

— tsdate

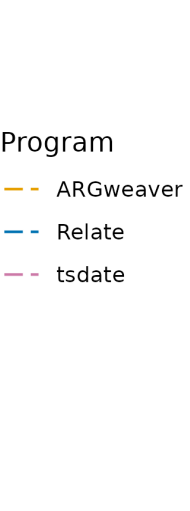

Program

- - ARGweaver

- - Relate

- - tsdate

Figure S2 Mean (A,B) and mean squared error (C,D) of point estimates of pairwise coalescence times by ARGweaver, Relate and tsdate in each bin of size 0.1 of simulated coalescence times. Diagonal gray line in plots $A$ and $B$ show 1:1 line. These results are for simulations with $n=8$ samples, mutation and recombination rates of $2 \times 10^{-8}$. Plots $B$ and $D$ are in log scale to highlight small values of coalescence times, which are the most abundant. Note that ARGweaver and Relate estimates are best (i.e. means in plots a and $b$ are closer to the simulated value) at values near the expected mean coalescence time under the coalescent (i.e. 1 in the coalescent units of $2 N_{e}$ generations). 
Theoretical vs. Simulated PDF

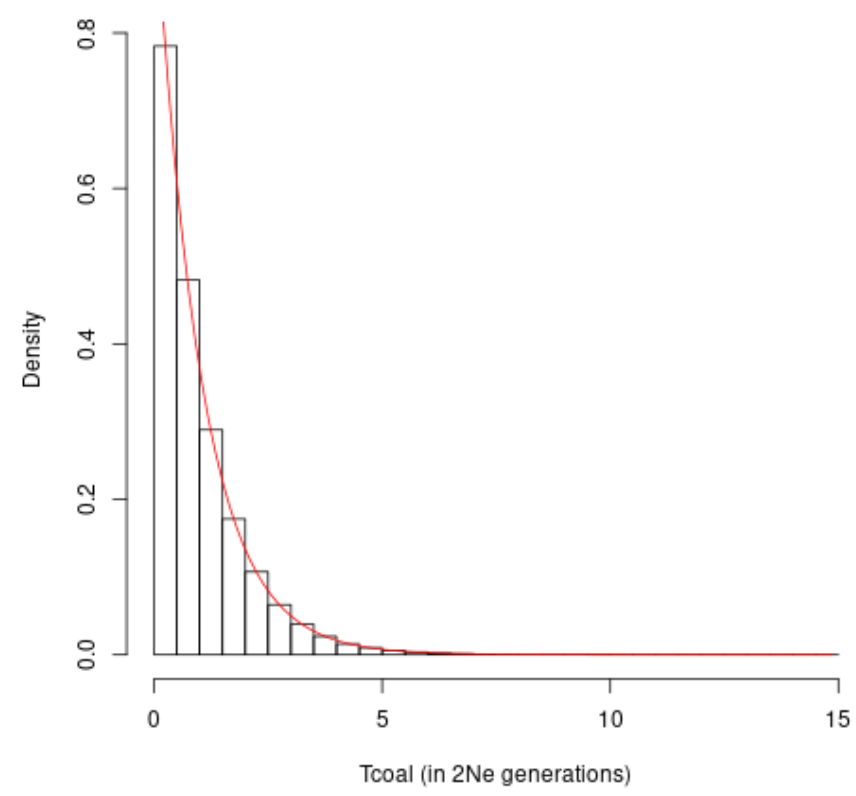

Figure S3 Histogram of the distribution of coalescence times in msprime simulations. Red line show expected exponential distribution with rate 1.

(a)

\section{Theoretical vs. Relate PM}

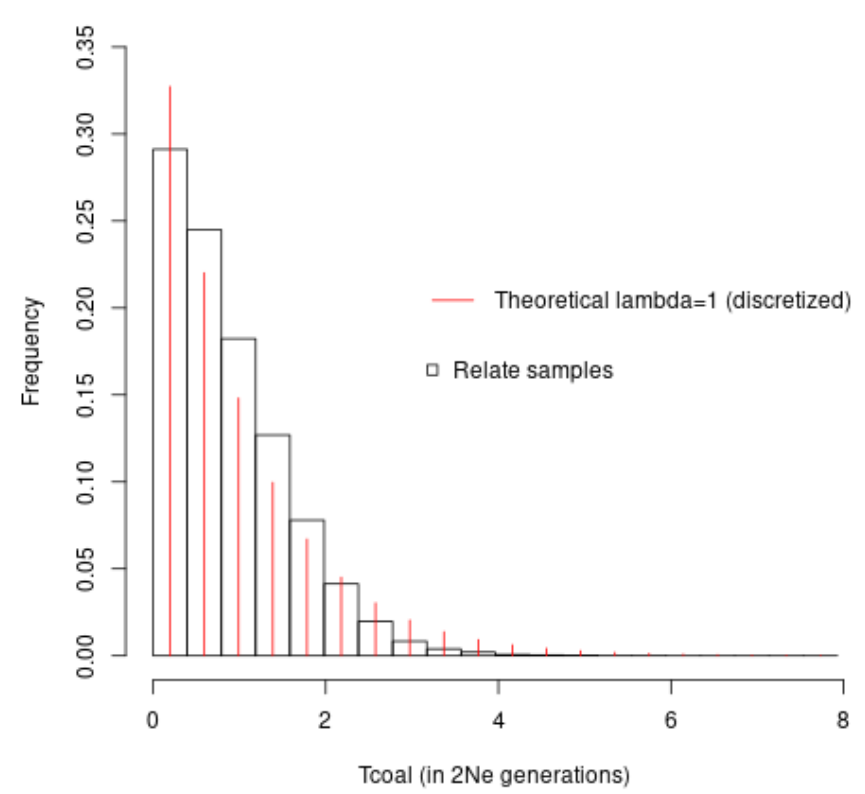

(b)

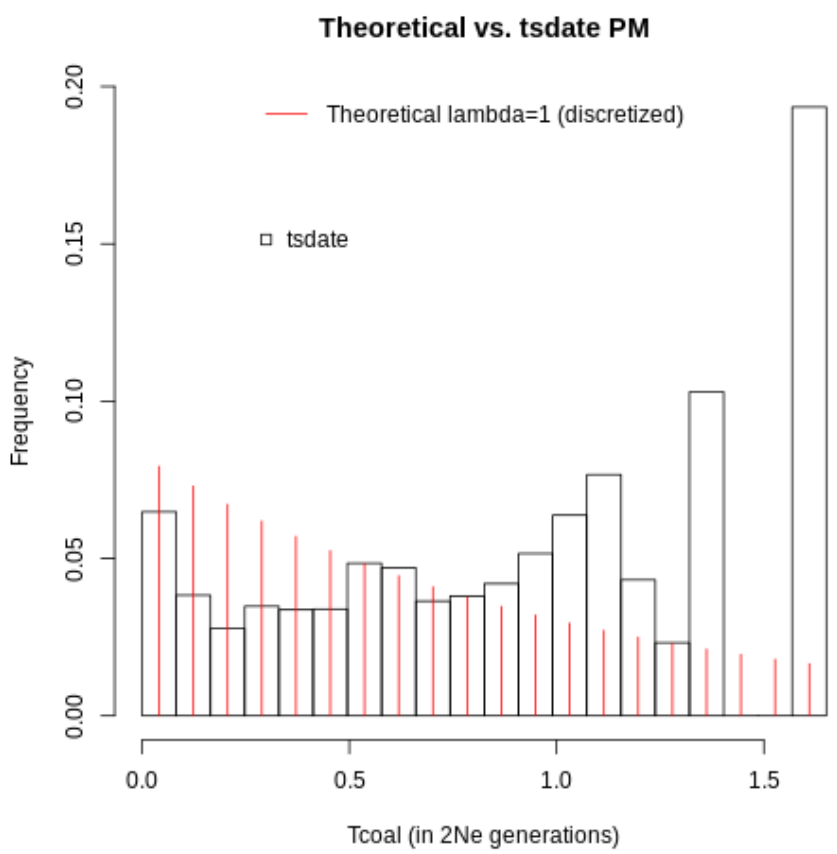

Figure S4 Distributions of pairwise coalescence times in Relate and tsdate without ARGweaver time discretization. These results are for simulations with $n=8$ samples, mutation and recombination rates of $2 \times 10^{-8}$. (a) Relate, (b) tsdate, both with 20 equal size bins 


\section{Evaluating MCMC Convergence}

To evaluate MCMC convergence in ARGweaver and Relate, we run these programs five independent times for the same simulated sequence of $5 \mathrm{Mb}$. We do this for each simulation scenario and evaluate convergence by analysing various statistics extracted at each iteration. For ARGweaver, we analyse statistics from in the stats file, described below. Relate does not generate a similar output, so we extract a subset of the pairwise coalescence times at each MCMC iteration to evaluate convergence. We also evaluate convergence based on selected pairwise coalescence times in ARGweaver, for comparison. Using these statistics extracted at each iteration, we evaluate MCMC convergence by analysing 1) trace plots, 2) autocorrelation plots, 3) effective sample sizes (Taboga 2017; Roy 2020), and 4) potential scale reduction factor (PSRF) (Gelman and Rubin 1992). Analyses and plots were done in $\mathrm{R}$ using the function acf for autocorrelation, and R package coda (Plummer et al. 2006) for effective sample sizes and potential scale reduction factor. These results were used to inform our decisions on burn-in and thinning for MCMC, as well as interpreting results of our evaluations of the methods under different simulated conditions.

\section{ARGweaver}

Convergence of likelihoods ARGweaver's arg-sample program outputs a stats file containing several statistics for each MCMC iteration: log probability of the sampled ARG given the model ("prior", in Table S1), log probability of the data given the sampled ARG ("likelihood"), total log probability of the ARG and the data ("joint"), number of recombination events in the sampled ARG ("recombs"), the number of variant sites that cannot be explained by a single mutation under the sampled ARG ("noncompats"), total length of all branches summed across sites ("arglen") (Hubisz and Siepel 2020). We generated trace plots and calculated autocorrelation between consecutive samples using the likelihood per iteration (Figures S5 and S6). Following visual inspection of these plots, we chose a burn-in consisting of the first 200 samples in most simulations, except in simulations with 10 times higher mutation rate (Figure S5C,F) or sample sizes larger than 8 haplotypes (Figure S6B,C,E,F), where we chose a burn-in of 1200 samples since those chains took longer to converge. In both cases, we ran MCMC for 1000 iterations after burn-in. Based on autocorrelation plots (Figure S5, S6) and on effective sample sizes (Table S1), we thinned ARGweaver samples by recording every 10th MCMC iteration, thus retaining a total of $100 \mathrm{MCMC}$ samples.

Results of the potential scale reduction factor suggested convergence of ARGweaver in simulations with mutation rate equal to recombination rate, with decreased recombination rate and with increased mutation rate (Table S1) - see section below on convergence of individual coalescence times.

Table S1 Potential scale reduction factor point estimates (PSRF), their upper confidence intervals (C.I.) and effective sample sizes $\left(N_{\text {eff }}\right)$ for ARGweaver stats. $\mu$ : mutation rate, $\rho$ : recombination rate.

\begin{tabular}{l|lll|lll|lll}
\hline & \multicolumn{3}{|c|}{$\mu=\rho \times 10^{-8}$} & \multicolumn{3}{|c|}{$\rho=2 \times 10^{-9}$} & \multicolumn{3}{l}{$\mu=2 \times 10^{-7}$} \\
& PSRF & C.I. & $N_{\text {eff }}$ & PSRF & C.I. & $N_{\text {eff }}$ & PSRF & C.I. & $N_{\text {eff }}$ \\
\hline prior & 1.06 & 1.15 & 224 & 1.01 & 1.03 & 494 & 1.00 & 1.01 & 216 \\
likelihood & 1.02 & 1.05 & 294 & 1.04 & 1.11 & 964 & 1.01 & 1.02 & 499 \\
joint & 1.06 & 1.16 & 216 & 1.01 & 1.02 & 486 & 1.01 & 1.02 & 219 \\
recombs & 1.04 & 1.1 & 254 & 1.01 & 1.03 & 559 & 1.00 & 1.01 & 229 \\
noncompats & 1.02 & 1.04 & 406 & 1.01 & 1.03 & 1290 & 1.01 & 1.04 & 518 \\
arglen & 1.06 & 1.16 & 348 & 1.08 & 1.21 & 459 & 1.05 & 1.12 & 319 \\
Multivariate & 1.15 & & & 1.1 & & & 1.05 & & \\
\hline
\end{tabular}


(a)

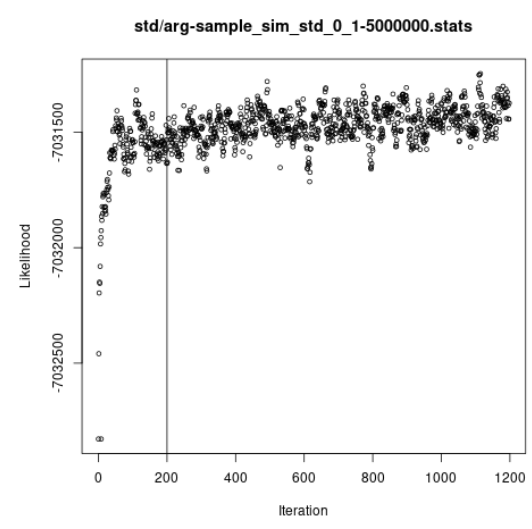

(d)

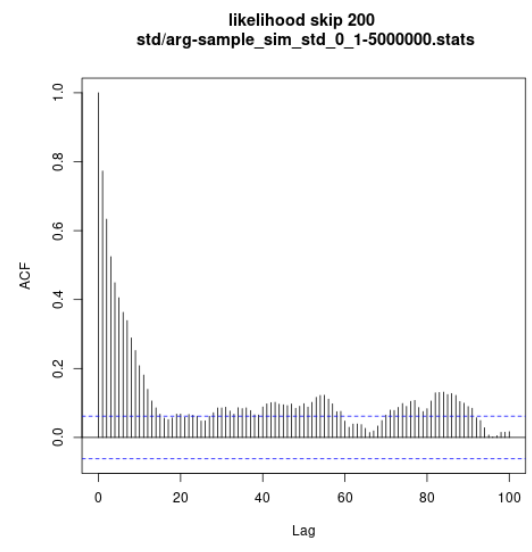

(b)

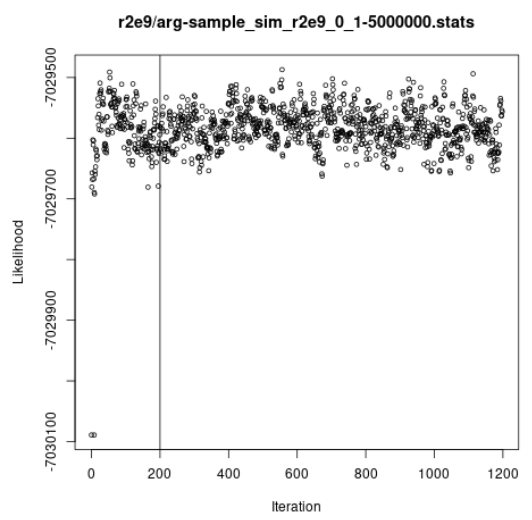

(e)

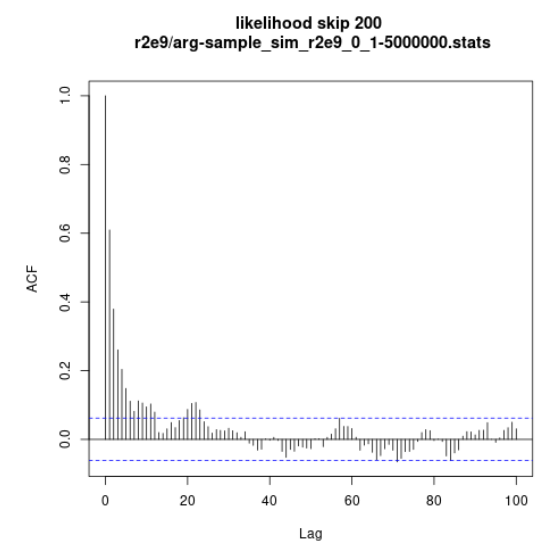

(c)

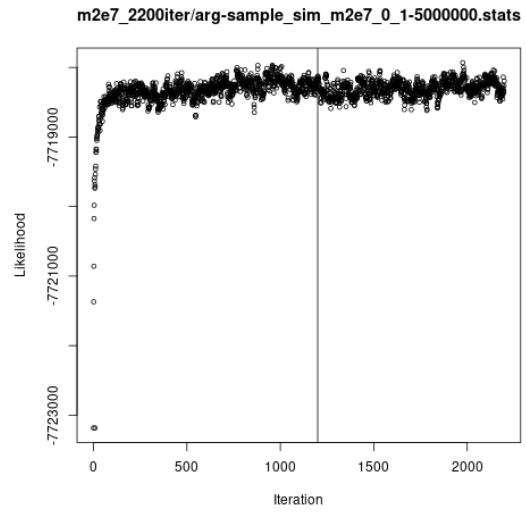

(f)

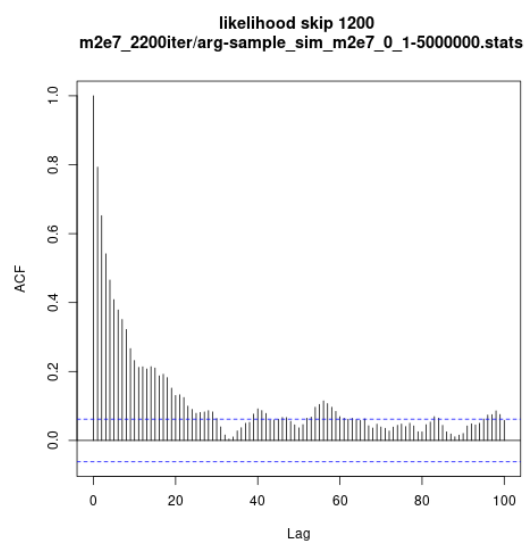

Figure S5 ARGweaver likelihood traces (top) and autocorrelation between consecutive MCMC iterations (bottom). A,D: simulations with 8 haplotypes, mutation rate equal to the recombination rate $\left(2 \times 10^{-8}\right)$. Potential scale reduction factor (PSRF) is 1.02, upper confidence interval (CI) is 1.05. B,E: simulations with recombination rate decreased to $2 \times 10^{-9}$. PSRF is 1.04 , upper CI is 1.11 . For both of these simulated datasets we used a burn in of 200 iterations (indicated by vertical line) and ran them for 1200 iterations in total, sampling every 10th iteration. C,F: simulations with mutation rate increased to $2 \times 10^{-7}$. PSRF is 1.01 , upper CI is 1.02. For this dataset we used a burn in of 1200 iterations (indicated by vertical line) and ran them for 2200 iterations in total, sampling every 10th iteration.

Convergence of coalescence times For comparison with Relate, which does not output statistics for each iteration, we also analyse convergence of pairwise coalescence times in ARGweaver. To this end, we extract from each MCMC iteration the values of coalescence times between two pairs of samples at 100 sites equally spaced by $50 \mathrm{~kb}$ along the $5 \mathrm{Mb}$ simulated sequences. We use those 200 values for convergence diagnostics. Figure S7 shows trace plots of 10 of those sites, for one pair of samples. To evaluate convergence, we calculate potential scale reduction factor (PSRF) for each of the 200 coalescence times, and compare their mean, variance and range (Table S2) among different simulations. In Table S2 we also compare the number of coalescence times that have effective sample sizes lower than 100 (which is our MCMC sample size). These results also lead us to conclude that ARGweaver runs with mutation rate equal to recombination rate have converged. However, in contrast to the results on convergence for statistics recorded in the ARGweaver stats files (Table S1), the evaluation of convergence based on coalescence times does not support a conclusion of full convergence for the other simulated data sets. In particular, simulations with mutation to recombination rate ratio of 10 had a large number of coalescence times with effective sample size smaller than 100 . The same was true for simulations with 16 and 32 haplotypes. The maximum values of PSRF in those simulations are also further from one, thus 
(a)

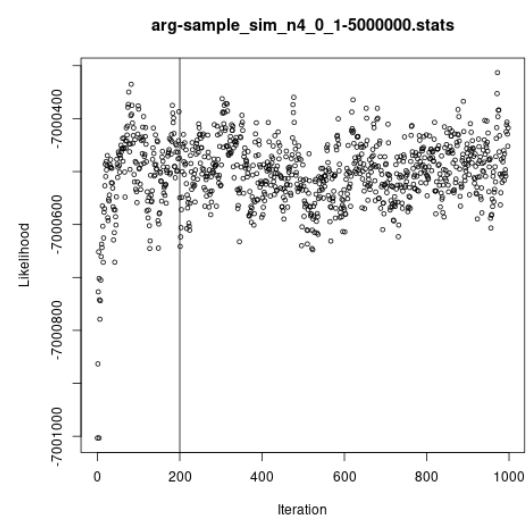

(d)

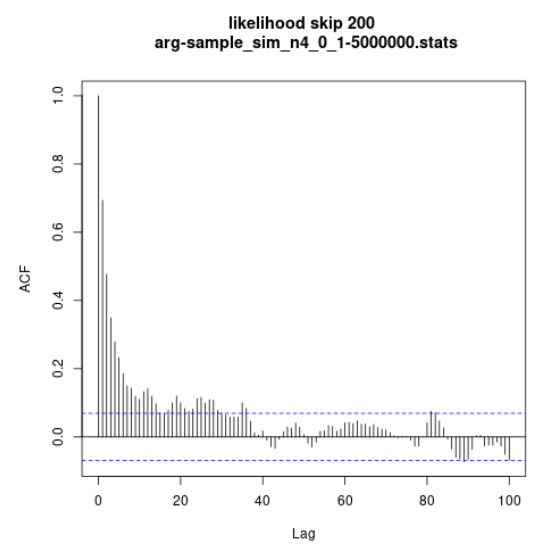

(b)

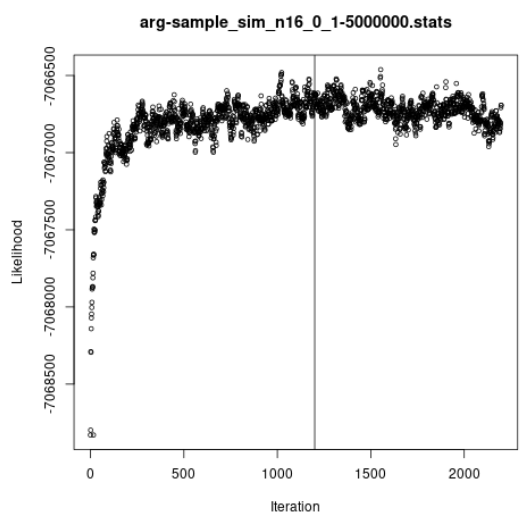

(e)

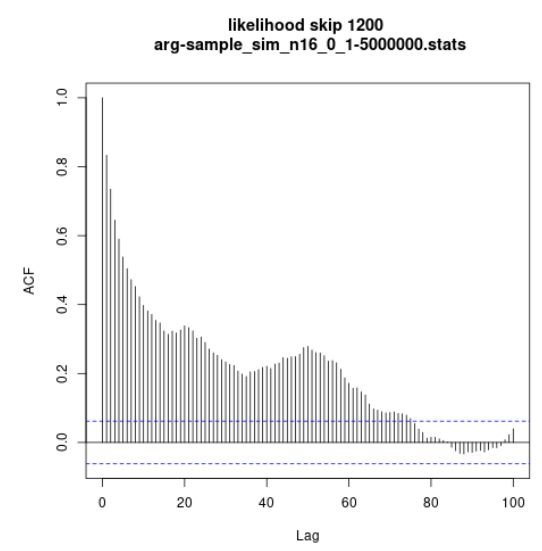

(c)

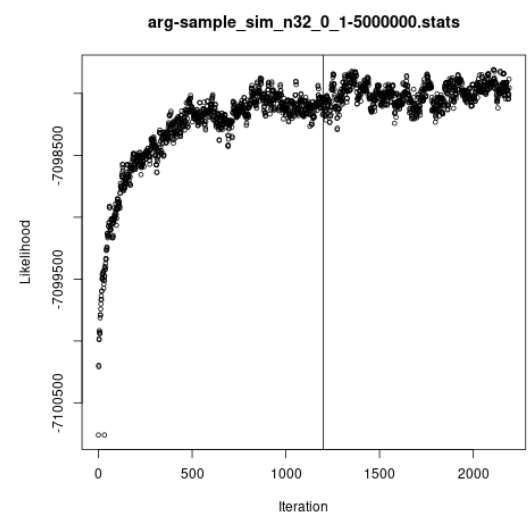

(f)

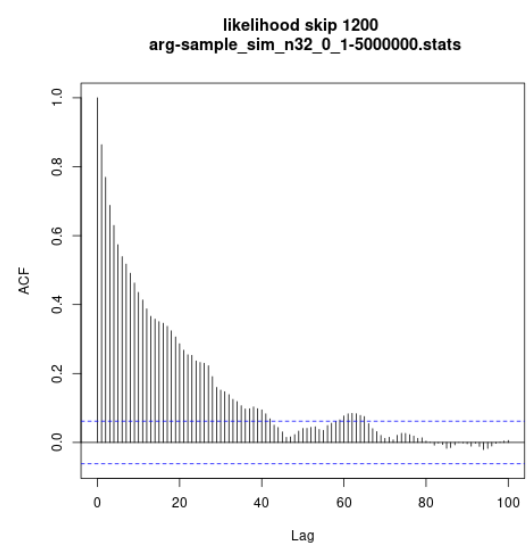

Figure S6 ARGweaver likelihood traces (top) and autocorrelation between consecutive MCMC iterations (bottom). A,D: simulations with 4 haplotypes, mutation rate equal to recombination rate $\left(2 \times 10^{-8}\right)$. For this simulated dataset we used a burn in of 200 iterations (indicated by vertical line) and ran them for 1200 iterations in total, sampling every 10th iteration. B,E: simulations with 16 haplotypes. C,F: simulations with 32 haplotypes. For both of these datasets we used a burn in of 1200 iterations (indicated by vertical line) and ran them for 2200 iterations in total, sampling every 10th iteration.

indicating a lack of convergence for some coalescence times.

Relate Relate estimates branch lengths using an MCMC algorithm with built in burn-in (Speidel et al. (2019) Supplementary Note on Method details 4.2, p. 13). To obtain samples from the posterior distribution, the tree sequence estimated in this first step was used as a stating point. Therefore, we did not implement any extra burn-in to obtain samples from the posterior. Visual inspection of traces plots also suggested that additional burn-in was not necessary (Figure S8).

We evaluated Relate's MCMC convergence by running it 5 times for each sequence of $5 \mathrm{Mb}$ simulated under each set of parameters, extracting a subset of pairwise coalescence times and calculating the potential scale reduction factor and effective sample sizes as described above for ARGweaver. Table S3 shows these results, which indicate convergence of all Relate runs in all simulated datasets. 
Table S2 Potential scale reduction factor (PSRF) mean, variance and range for each of 200 coalescence times in ARGweaver, the multivariate PSRF (Plummer et al. 2006) and the number of coalescence times for each the effective sample size $\left(N_{e f f}\right)$ is smaller than 100 . Unless otherwise noted, mutation rate $(\mu)$ and recombination rate $(\rho)$ are $2 \times 10^{-8}$ and sample sizes $(n)$ are 8 haplotypes.

\begin{tabular}{l|llllll}
\hline PSRF & $\mu=\rho$ & $\rho=2 \times 10^{-9}$ & $\mu=2 \times 10^{-7}$ & $\mathrm{n}=4$ & $\mathrm{n}=16$ & $\mathrm{n}=32$ \\
\hline Mean & 1.055 & 1.069 & 1.211 & 1.028 & 1.242 & 244.152 \\
Variance & 0.005 & 0.010 & 1.053 & 0.001 & 0.233 & 11613699 \\
& $0.994-$ & $0.994-$ & $0.994-$ & $0.991-$ & $1.001-$ & $0.994-$ \\
Range & 1.415 & 1.709 & 13.740 & 1.199 & 4.847 & $4.783 \times 10^{4}$ \\
& 4.92 & 4.29 & 21.2 & 2.78 & 24.9 & 110560 \\
Multivariate & 4.92 & & & & \\
Number of & & & 14 & 0 & 32 & 45 \\
$N_{\text {eff }}<100$ & 4 & 16 & & & & \\
(out of 200) & & & & & &
\end{tabular}

Table S3 Potential scale reduction factor (PSRF) mean, variance and range for each of 200 coalescence times in Relate, the multivariate PSRF (Plummer et al. 2006) and the number of coalescence times for each the effective sample size $\left(N_{e f f}\right)$ is smaller than 100. Unless otherwise noted, mutation rate $(\mu)$ and recombination rate $(\rho)$ are $2 \times 10^{-8}$ and sample sizes $(n)$ are 8 haplotypes.

\begin{tabular}{l|llllll}
\hline PSRF & $\mu=\rho$ & $\rho=2 \times 10^{-9}$ & $\mu=2 \times 10^{-7}$ & $\mathrm{n}=4$ & $\mathrm{n}=16$ & $\mathrm{n}=32$ \\
\hline Mean & 1.007 & 1.007 & 1.008 & 1.008 & 1.009 & 1.008 \\
Variance & $10^{-4}$ & $8.7 \times 10^{-5}$ & $10^{-4}$ & $10^{-4}$ & $10^{-4}$ & $10^{-4}$ \\
& $0.991-$ & $0.993-$ & $0.992-$ & $0.991-$ & $0.992-$ & $0.993-$ \\
Range & 1.076 & 1.051 & 1.051 & 1.102 & 1.061 & 1.049 \\
& 2.24 & 2.12 & 2.57 & 2.24 & 3.31 & 2.49 \\
Multivariate & 2.24 & & & & \\
Number of & & & 0 & 0 & 0 & 0 \\
$N_{\text {eff }}<100$ & 0 & 0 & & & &
\end{tabular}


bioRxiv preprint doi: https://doi.org/10.1101/2021.11.15.468686; this version posted November 27, 2021. The copyright holder for this preprint (which was not certified by peer review) is the author/funder, who has granted bioRxiv a license to display the preprint in perpetuity. It is made available under aCC-BY-NC 4.0 International license.

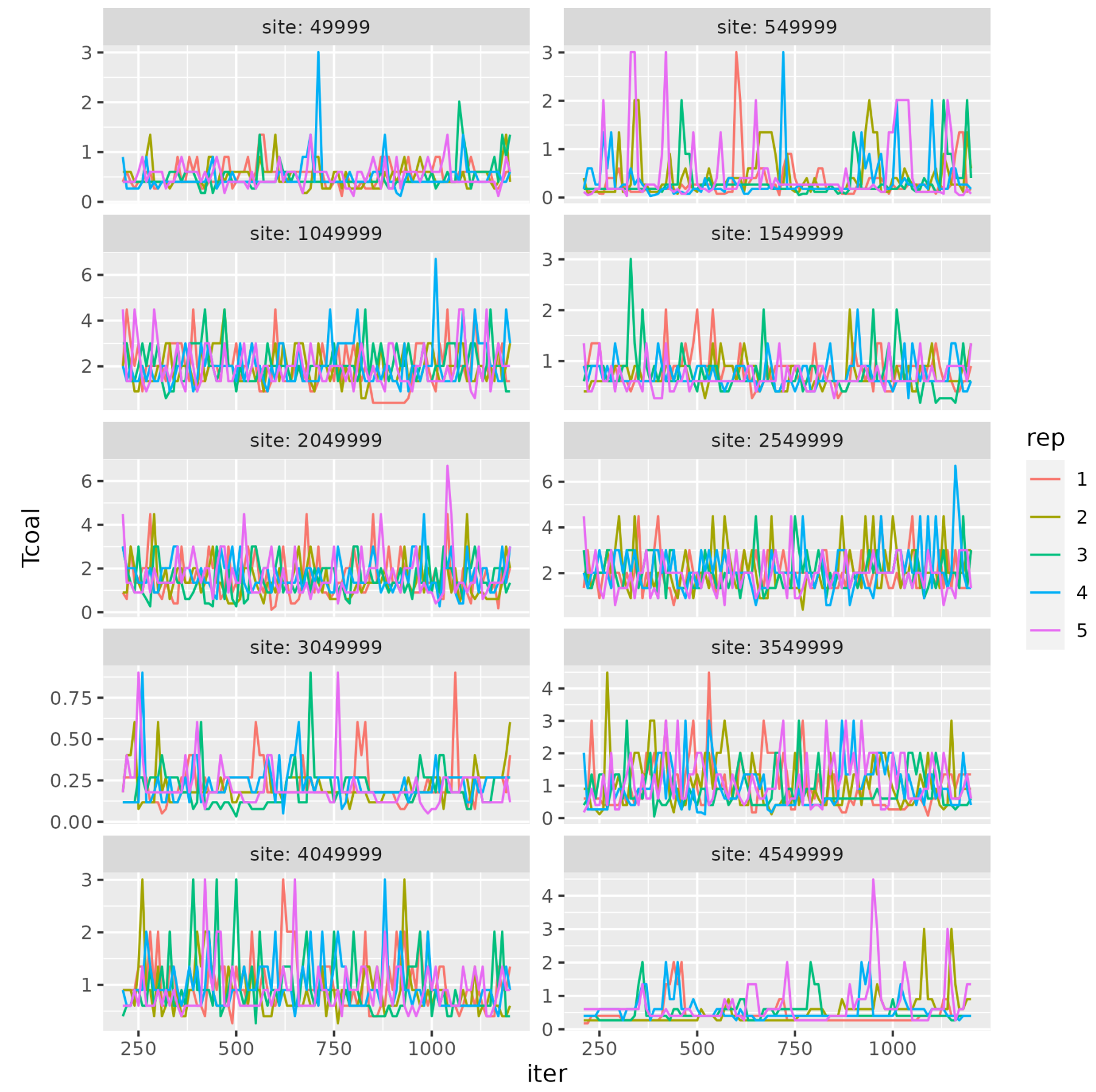

Figure $\mathbf{S 7}$ Coalescence times for one pair of samples inferred by 5 independent runs of ARGweaver at 10 sites equally spaced sites along the $5 \mathrm{Mb}$ sequence. Simulations with 8 samples and mutation rate equal to recombination rate. 
bioRxiv preprint doi: https://doi.org/10.1101/2021.11.15.468686; this version posted November 27, 2021. The copyright holder for this preprint (which was not certified by peer review) is the author/funder, who has granted bioRxiv a license to display the preprint in perpetuity. It is made available under aCC-BY-NC 4.0 International license.
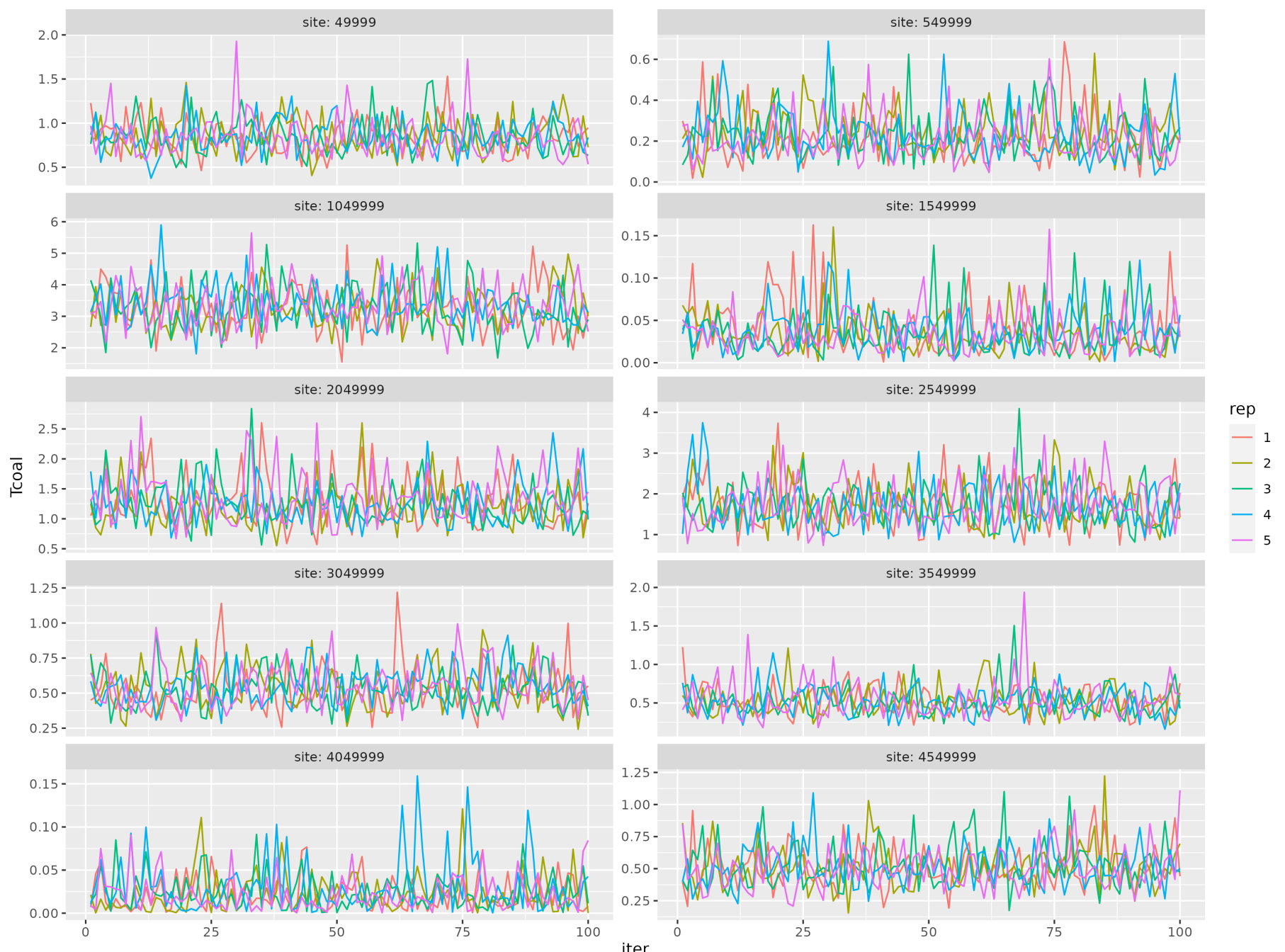

Figure $\mathbf{S 8}$ Coalescence times for one pair of samples inferred by 5 independent runs of Relate at 10 sites equally spaced sites along the $5 \mathrm{Mb}$ sequence. Simulations with 8 samples and mutation rate equal to recombination rate. 


\section{Additional simulations results for ARGweaver}

SMC and SMC' modes in ARGweaver In all results shown in the main text, we simulated under the standard Hudson (1983) coalescent with recombination, and did inference in ARGweaver under SMC'. Here, we asked whether deviations observed in the posterior distribution of ARGweaver can be explained by differences between the models used for simulation and inference. For this, we simulate sequences in msprime under the SMC and SMC' models, and run ARGweaver inference using the same model used in the simulation. We simulated 8 haplotypes with mutation rate and recombination rate $2 \times 10^{-8}$. Results improve when simulating under SMC' and inferring under SMC' (Figures S9B, S10B). Surprisingly, simulating and inferring under SMC (Figures S9A, S10A) is not better than simulating under the full coalescent with recombination model and inferring under SMC (Figures 3,4).

(a) SMC

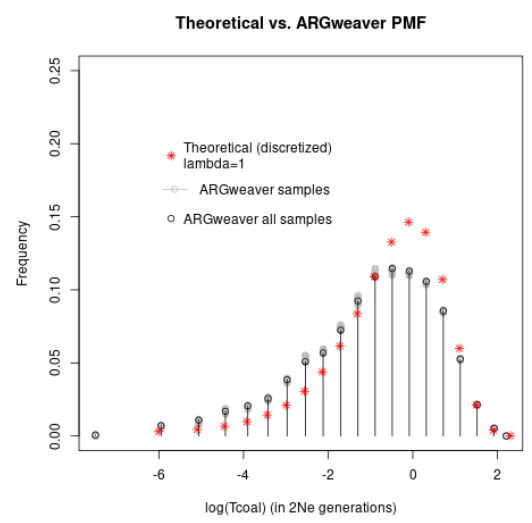

(b) $S M C^{\prime}$

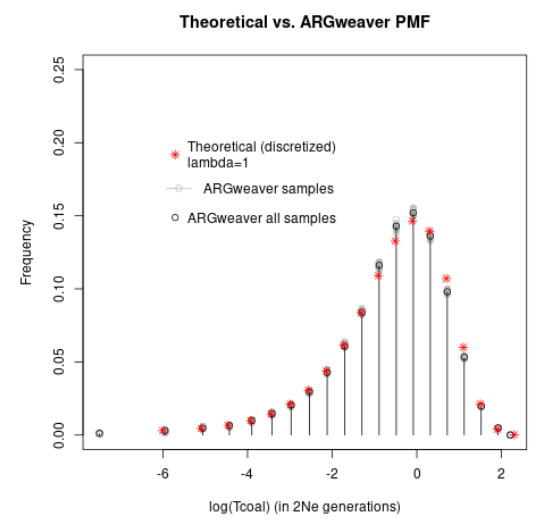

Figure S9 Distribution of coalescence times in msprime simulations using the SMC (A) or SMC' model (B). ARGweaver inference is done using the same model used in the simulations.

(a) SMC

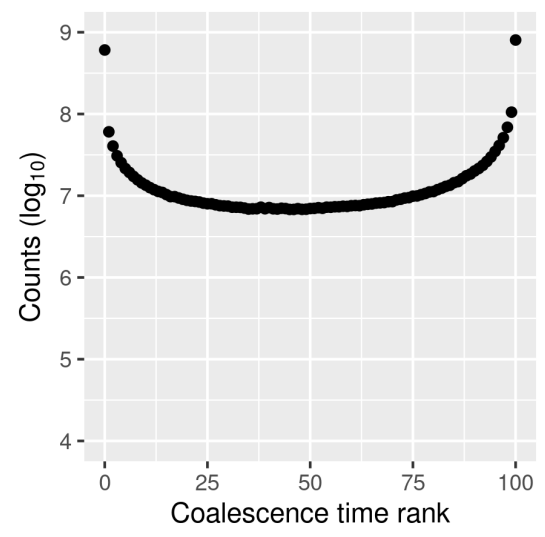

(b) $\mathrm{SMC}^{\prime}$

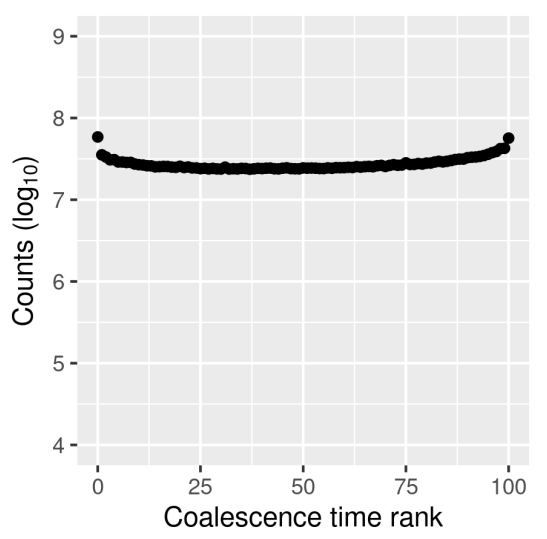

Figure S10 Simulation-based calibration results in msprime simulations using the SMC (A) or SMC' model (B). ARGweaver inference is done using the same model used in the simulations. 
(a)

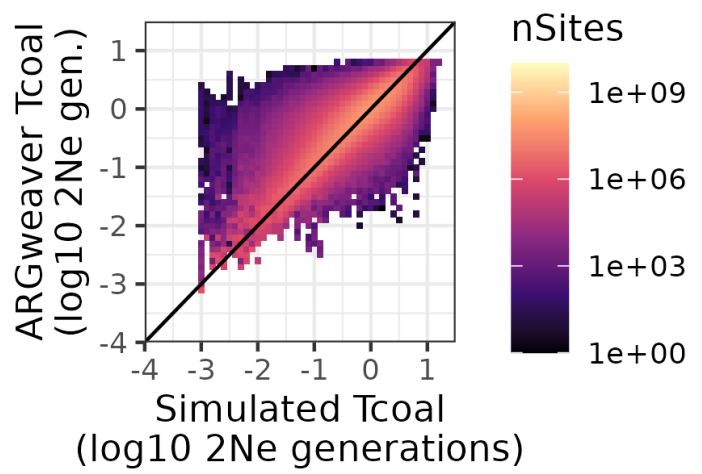

(d)

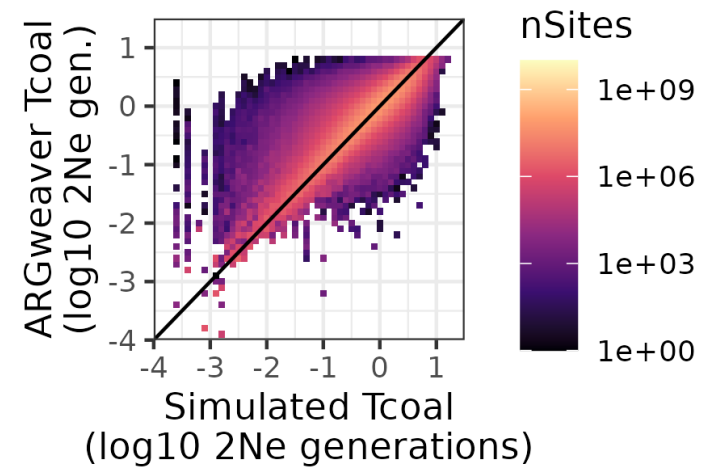

(b)

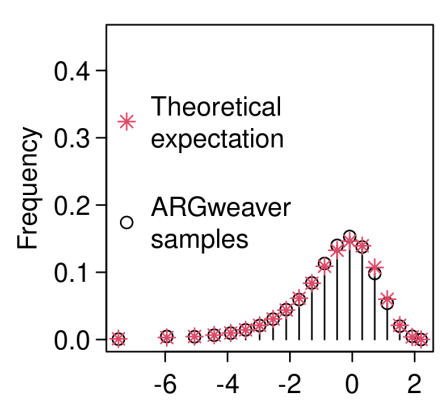

$\log ($ Tcoal) (in 2Ne generations)

(e)

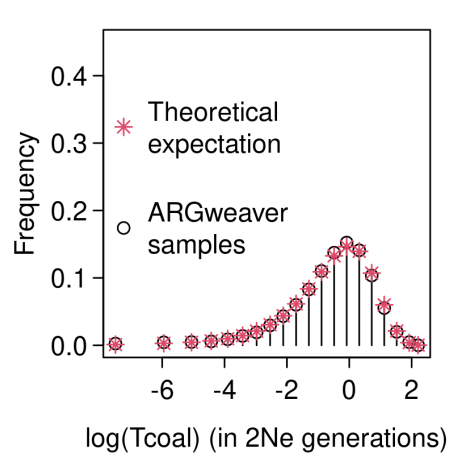

(c)

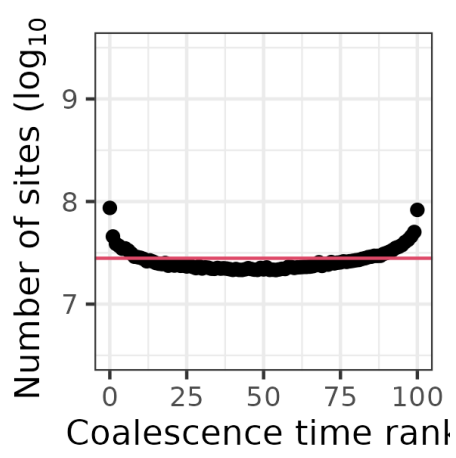

(f)

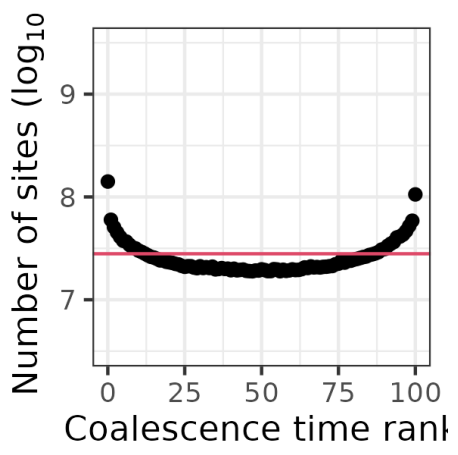

Figure S11 Evaluation of ARGweaver point estimates (A,D), distribution of coalescence times (B,E) and posterior calibration $(\mathrm{C}, \mathrm{F})$ for simulations with mutation rate to recombination rate ratio of $2(\mathrm{~A}-\mathrm{C}, \mu=$ $4 \times 10^{-8}, \rho=2 \times 10^{-8}$ ) and mutation rate to recombination rate ratio of 4 (D-F, $\mu=8 \times 10^{-8}, \rho=2 \times 10^{-8}$ )

Intermediate values of mutation to recombination rate ratio Rasmussen et al. (2014) mention in their Figure S5 that the quality of ARGweaver estimates generally improved in their simulations with increased mutation to recombination rates ratio $(\mu / \rho)$, but only up to $\mu / \rho=4$. Motivated by this observation, we additionally ran simulations with values of $\mu / \rho$ in between the ones shown in the main text $(\mu / \rho=1$ or $\mu / \rho=10)$, including $\mu / \rho=2$ and 4 . We summarize our results under these conditions in Table S4. We observed a similar pattern for these intermediate values of $\mu / \rho=2,4$ as we had observed from 1 to 10, i.e. point estimates improve with increased ratio (shown by lower MSE in Table S4), and calibration of the posterior distribution worsens with an increased ratio (show by higher KLD in Table S4). 
Jukes-Cantor mutational model In all results shown in the main text, we simulated mutations using an infinite sites model. ARGweaver, on the other hand, uses a Jukes and Cantor (1969) mutational model. Therefore, we hypothesize that differences in the mutational model between simulations and inference could explain deviations in the posterior distribution of ARGweaver, especially in simulations with increased mutation to recombination ratio $(\mu / \rho)$. We found that ARGweaver results with simulations under the Jukes and Cantor (1969) model are very similar to the results under the infinite sites model and follow the same pattern under increased $\mu / \rho$ (Table S4, Figures S12, S13).

Table S4 Comparison of ARGweaver results with simulations under infinite sites mutational model and Jukes-Cantor finite sites mutational model, including simulations with values of mutation to recombination rate ratio in between the ones shown in the main text. ${ }^{*}$ indicate results shown in the main text and presented here again for comparison.

\begin{tabular}{c|cc|cc}
\hline & \multicolumn{2}{|c|}{$\begin{array}{c}\text { Point estimates (MSE) } \\
\text { Ranks (KLD) }\end{array}$} & \multicolumn{2}{c}{ Rites } \\
$\mu / \rho$ & Infinite sites & Finite sites (JC) & Infinite sites & Finite sites (JC) \\
\hline$\frac{2 \times 10^{-8}}{2 \times 10^{-8}}=1$ & $0.397^{*}$ & 0.396 & $0.027^{*}$ & 0.026 \\
$\frac{4 \times 10^{-8}}{2 \times 10^{-8}}=2$ & 0.285 & 0.285 & 0.049 & 0.053 \\
$\frac{8 \times 10^{-8}}{2 \times 10^{-8}}=4$ & 0.195 & 0.197 & 0.113 & 0.112 \\
$\frac{2 \times 10^{-7}}{2 \times 10^{-8}}=10$ & $0.117^{*}$ & 0.120 & $0.350^{*}$ & 0.353 \\
$\frac{2 \times 10^{-8}}{2 \times 10^{-9}}=10$ & $0.120^{*}$ & 0.119 & $0.286^{*}$ & 0.291 \\
\hline
\end{tabular}


(a)

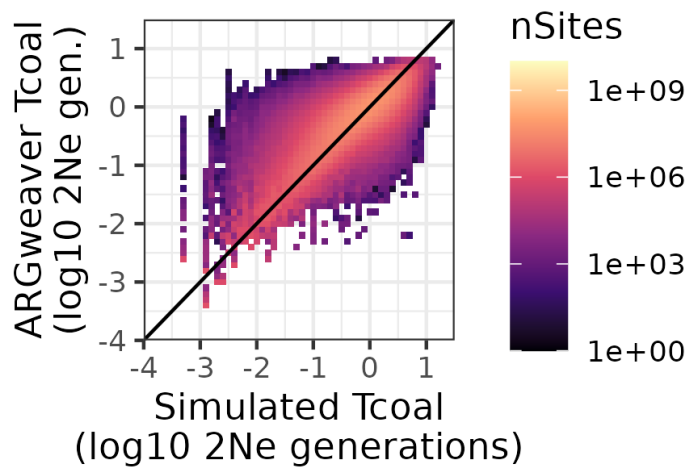

(d)

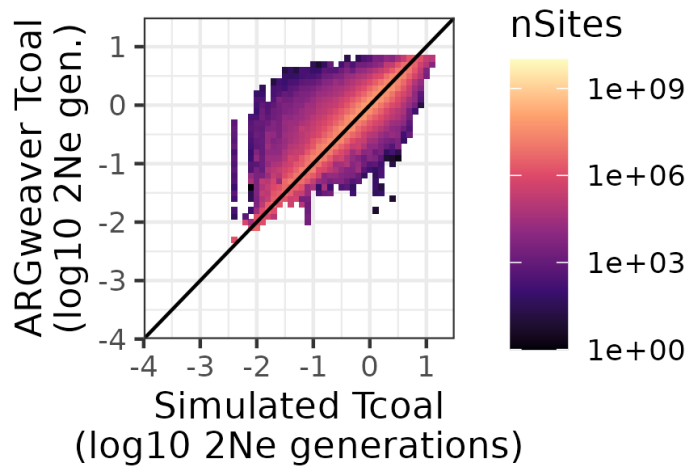

(b)

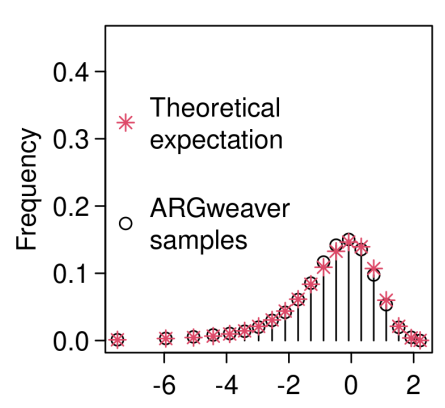

$\log$ (Tcoal) (in 2Ne generations)

(e)

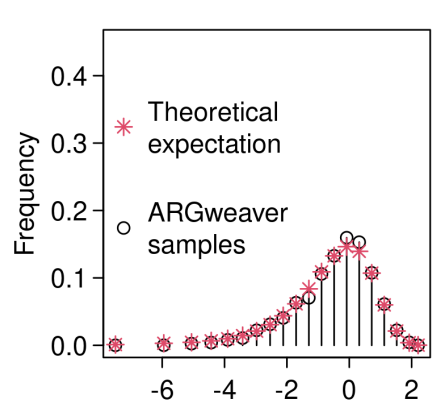

$\log$ (Tcoal) (in 2Ne generations) (c)

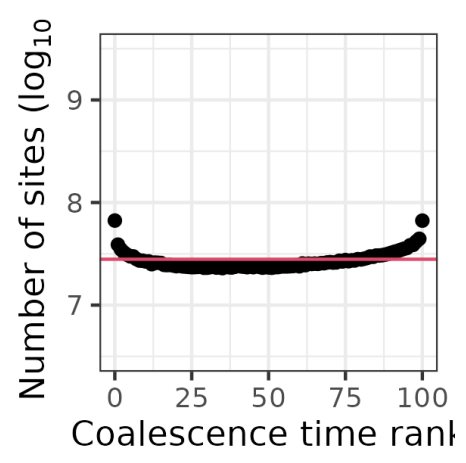

(f)

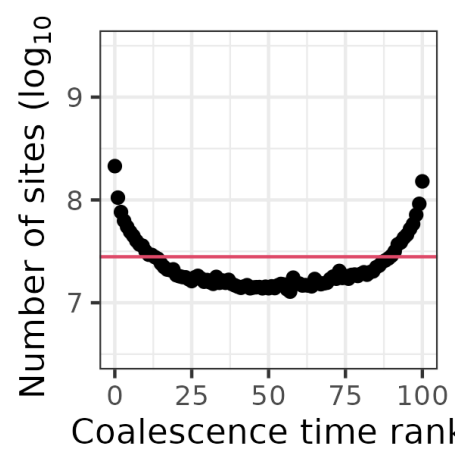

Figure S12 Evaluation of ARGweaver point estimates (A,D), distribution of coalescence times (B,E) and posterior calibration (C,F) with simulations under the Jukes and Cantor (1969) mutational model. A-C: simulations with 8 haplotypes and $\mu=\rho=2 \times 10^{-8}$. D-F: simulations with 8 haplotypes and $\mu=2 \times 10^{-8}$ and $\rho=2 \times 10^{-9}$ 
(a)

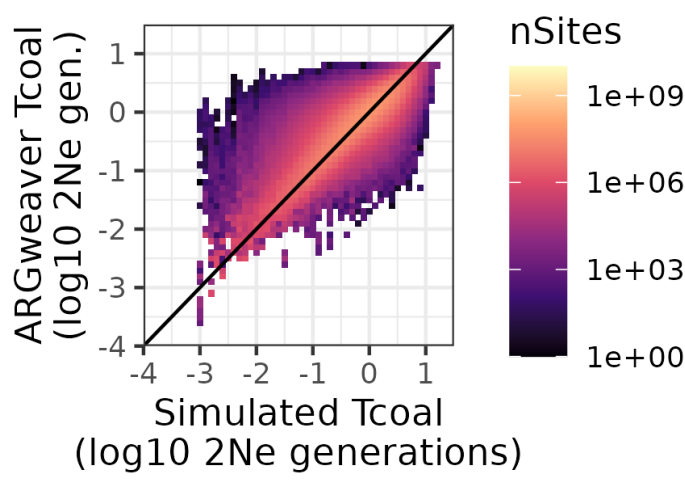

(d)

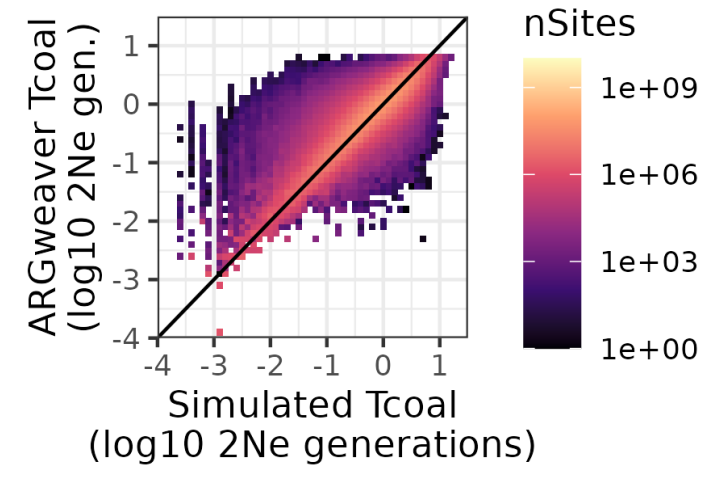

(g)

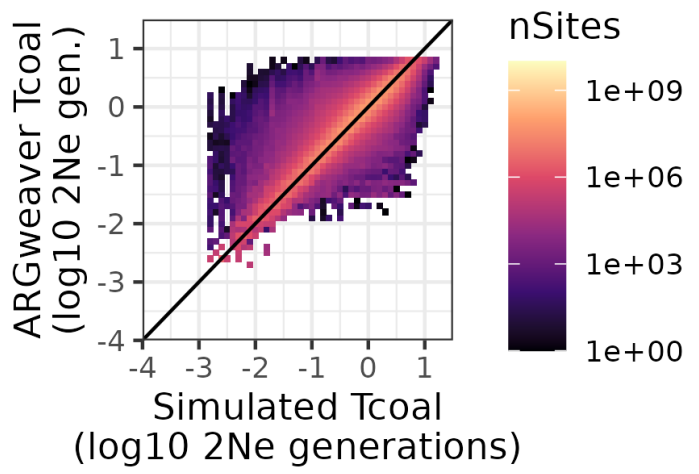

(b)

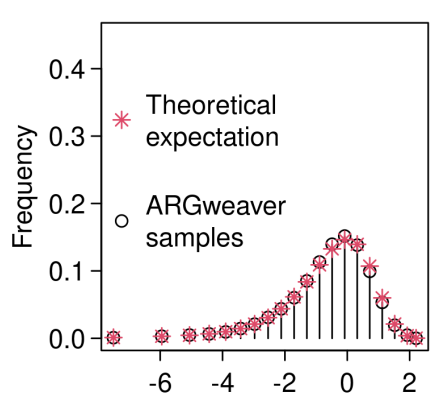

$\log ($ Tcoal) (in 2Ne generations)

(e)

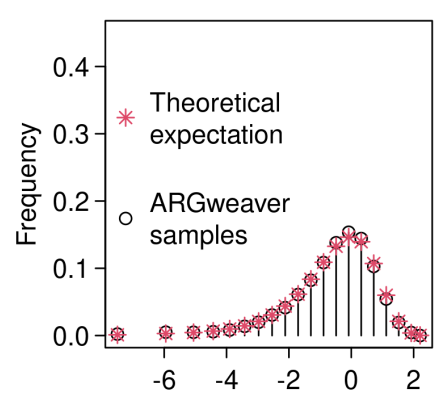

$\log$ (Tcoal) (in 2Ne generations)

(h)

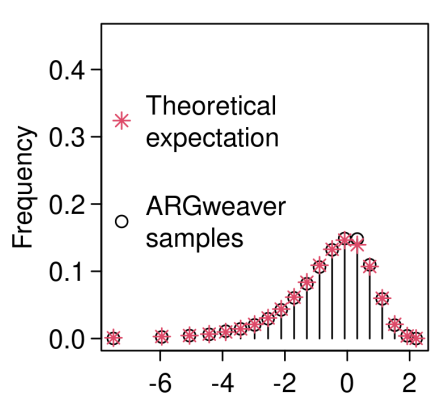

$\log ($ Tcoal) (in 2Ne generations) (c)

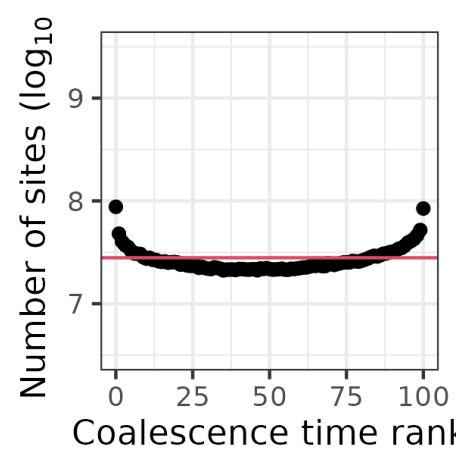

(f)

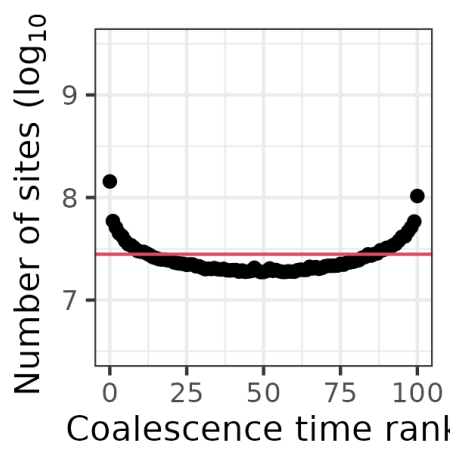

(i)

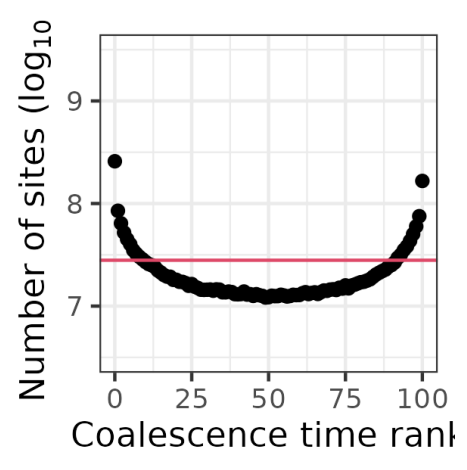

Figure S13 Evaluation of ARGweaver point estimates (A,D,G), distribution of coalescence times (B,E,H) and posterior calibration (C,F,I) with simulations under the Jukes and Cantor (1969) mutational model. In all cases we simulated 8 haplotypes and used $\rho=2 \times 10^{-8}$. A-C: $\mu=4 \times 10^{-8}$. D-F: $\mu=8 \times 10^{-8}$. G-I: $\mu=2 \times 10^{-7}$ 